\title{
Homogenization of the wave equation with non-uniformly oscillating coefficients
}

\author{
Danial P. Shahraki, Bojan B. Guzina* \\ Department of Civil, Environmental and Geo- Engineering, University of Minnesota, Twin Cities, MN 55455, USA
}

\begin{abstract}
The focus of our work is dispersive, second-order effective model describing the low-frequency wave motion in heterogeneous (e.g. functionally-graded) media endowed with periodic microstructure. For this class of quasiperiodic medium variations, we pursue homogenization of the scalar wave equation in $\mathbb{R}^{d}, d \geqslant 1$ within the framework of multiple scales expansion. When either $d=1$ or $d=2$, this model problem bears direct relevance to the description of (anti-plane) shear waves in elastic solids. By adopting the lengthscale of microscopic medium fluctuations as the perturbation parameter, we synthesize the germane low-frequency behavior via a fourth-order differential equation (with smoothly varying coefficients) governing the mean wave motion in the medium, where the effect of microscopic heterogeneities is upscaled by way of the so-called cell functions. In an effort to demonstrate the relevance of our analysis toward solving boundary value problems (deemed to be the ultimate goal of most homogenization studies), we also develop effective boundary conditions, up to the second order of asymptotic approximation, applicable to one-dimensional (1D) shear wave motion in a macroscopically heterogeneous solid with periodic microstructure. We illustrate the analysis numerically in 1D by considering (i) low-frequency wave dispersion, (ii) mean-field homogenized description of the shear waves propagating in a finite domain, and (iii) full-field homogenized description thereof. In contrast to (i) where the overall wave dispersion appears to be fairly well described by the leading-order model, the results in (ii) and (iii) demonstrate the critical role that higher-order corrections may have in approximating the actual waveforms in quasi-periodic media.
\end{abstract}

Keywords: dynamic homogenization, waves, quasi-periodic media, effective boundary conditions

\section{Introduction}

Making use of phenomena such as dispersion, frequency-dependent anisotropy, band gaps, frequency-selective reflection, and negative index of refraction [12, 1, 2, phononic materials and periodic composites can be tailored in a way to manipulate waves toward achieving cloaking, vibration control, and sub-wavelength imaging [36, 49, 34]. Simulating the underpinning wave motion problem that features rapid (sub-wavelength)

\footnotetext{
${ }^{*}$ Corresponding Author: Bojan B. Guzina; 500 Pillsbury Drive SE, Minneapolis, MN 55455; Email: guzin001@umn.edu; Phone: 612-626-0789.
} 
variations in the medium, however, can be computationally taxing. To alleviate the impediment, one idea is to "homogenize" the medium, i.e. to obtain an effective field equation for the problem that simultaneously: (i) captures salient attributes of the generated wave motion, and (ii) features "smoothly-varying" (typically constant) coefficients that are devoid of rapid variations.

There is a vast body of literature on the homogenization of wave motion in periodic media. One school of thought - that is rooted in engineering mechanics and targets an effective description of composites - is the Willis' method of effective constitutive relationships [36, 37, 40, 35]. Another keen approach to obtaining the "macroscopic" description of periodic media is based on the Floquet-Bloch theory [28, 10, 12 , which considers the problem eigenfunctions in the form of a plane wave modulated by a periodic function [e.g. 43, 41. This method is often used to obtain the inherent (multi-valued) dispersion relationship, including band gaps, for periodic media. The third school of thought, rooted in mathematics [5, 9, 42, 6, is the method of multiple scales expansion where the perturbation parameter is defined as the vanishing ratio between the lengthscale of medium fluctuations and some finite wavelength.

When multiple-scales homogenization is deployed to describe waves in periodic media, the underpinning asymptotic expansion translates the governing differential equation with rapidly-oscillating coefficients into that with constant coefficients, featuring powers of the perturbation parameter. If the homogenization ansatz is pursued to the leading order only, one obtains the classical homogenization theory and "effective" medium properties, for instance the effective elastic moduli and effective mass density in the context of elastodynamics. To extend the dynamic range of such "long-wavelength" model, higher-order corrections - representing singular perturbations of the leading-order effective field equation - bring about the effects of incipient dispersion and frequency-dependent medium anisotropy. Two-scale homogenization of the wave equation in periodic structures is nowadays well understood. For instance, higher-order asymptotic expansions of the low-frequency (and thus long-wavelength) wave motion in periodic media were studied in one [24, 17, 26, 8, and multiple [11, 27, 25, 7, 4, 46, spatial dimensions.

To better manipulate waves for the purposes of e.g. vibration isolation or energy harvesting, however, one may also consider introducing global (i.e. macroscopic) medium variations that may act in concert with their microscopic counterpart. One such example is the concept of rainbow trapping [e.g. 45, 48, where an arrangement of dissimilar unit cells - say sub-wavelength "resonators" with progressively varying dynamic characteristics - is used to enlarge a band gap, relative to what is achievable by purely periodic assemblies. In this setting, it is useful to think of a functionally-graded medium 38 endowed with periodic microstructure, see for example the "staircase" profiles [48, 18] designed to rainbow trap acoustic and seismic waves. A recent study [16] further demonstrates the spatial arrangement of a given spectrum of unit cells may significantly affect the performance of a rainbow trap. In the context of optimal design, this exposes the need for rationally constructing a homogenized description of media whose material properties vary on both macroscopic and microscopic scales. As will be seen shortly, our study investigates the low-frequency behavior of such media 
and does not cover the phenomenon of rainbow trapping; however a generalization of this work in the context of high-frequency homogenization [20] could find immediate use in the optimal design of such "band-stop" filters.

In the literature, a limited number of works have addressed the homogenization of media with both macroand micro-scale variations, especially when considering higher-order asymptotic corrections. Among the earliest studies, one can refer to the multiple-scales frameworks [9, 6] that (among other topics) consider elliptic boundary value problems for equations with non-uniformly oscillating coefficients. In [3], a novel asymptotic approach was introduced to integrate differential equations governing quasi-periodic structures. The common thread in these developments is their focus on the leading-order homogenized model. Transcending such limitation, a first-order multiscale expansion of the linear elasticity problem for quasi-periodic structures was investigated in [14]. More recently, [23, 44, 33, 47] considered a second-order multiple scales description of the heat conduction, linear elasticity, and thermo-elastic problems in quasi-periodic porous materials. A second-order macroscopic elastic energy of the quasi-periodic materials was investigated in 32 .

Concerning the wave motion in quasi-periodic media, homogenization of the elastic i.e. seismic wave equation was pursued via multiple scales expansion up to the leading i.e. zeroth order for three-dimensional waves [21], and up to the first order for two-dimensional (anti-plane shear) SH and (in-plane compressional and shear) P-SV waves [30, 15]. Recently, 22] proposed a second-order, two-scale asymptotic model of the damped wave equation in quasi-periodic media.

At this point, however, one should note that the first- and second-order models in [30, 15, 22] are incomplete for they disregard asymptotic corrections of the mean wave motion. In this vein, the authors in [30, 15] refer to their expansions as those of "partial order one". To highlight the issue, let us denote the featured wave motion by $u$, its mean-field variation by $\langle u\rangle$, and the germane perturbation parameter by $\epsilon$. In this setting, the multiple-scales approach results in an asymptotic expansion

$$
u(x)=u_{0}(x)+\epsilon u_{1}(x, y)+\epsilon^{2} u_{2}(x, y)+\ldots
$$

where $x$ and $y=\epsilon^{-1} x$ on the right-hand side signify the so-called "slow" and "fast" variable, respectively, while the asymptotic corrections $u_{j}(x, y)$ are built recursively in terms of: (i) lower-order mean-field variations $\left\langle u_{k}\right\rangle(x), k=\overline{0, j-1}$ that are governed by the respective (homogenized) field equations, and (ii) fastoscillating cell functions that depend exclusively on medium properties. In periodic media, it is well known [e.g. 39, 46] that $\left\langle u_{1}\right\rangle \equiv 0$. In quasi-periodic media, on the other hand, $\left\langle u_{1}\right\rangle \neq 0$ in general. As a result, discarding $\left\langle u_{j}\right\rangle, j \geqslant 1$ as in $[30$, 15, 22] leads to incomplete i.e. "partial" higher-order solutions.

In this vein, we pursue a (complete) second-order homogenization of the scalar wave equation in $\mathbb{R}^{d}$, $d \geqslant 1$ for the class of quasi-periodic media that feature: (i) smooth macroscopic variation, and (ii) periodic microscopic fluctuation. The analysis commences with a one-dimensional primer $(d=1)$ and demonstrates, via the multiple scales approach, that second-order homogenization of low-frequency wave motion in a medium with non-uniformly (yet "rapidly") oscillating coefficients yields a fourth-order effective differential equation 
with smoothly varying coefficients. This result is then extended to describe the effective wave motion in quasi-periodic media for $d>1$. Motivated by a recent (one-dimensional) study of the effective wave motion in bounded periodic domains [19], we next develop effective "Dirichlet" and "Neumann" boundary conditions (up to the second order of asymptotic approximation) for one-dimensional waves in quasi-periodic media, and we use this result to homogenize one-dimensional boundary value problem for a quasi-periodic domain of finite extent. A set of numerical results including (i) dispersion curves, (ii) mean-filed approximations, and (iii) full-field approximations of the wave motion in quasi-periodic media is included to illustrate the utility of the proposed homogenization framework. In contrast to (i) where the overall wave dispersion (due to combined "action" of micro- and macro-scale heterogeneities) appears to be well described by the leading-order model, the results in (ii) and (iii) highlight the critical role that higher-order corrections have in maintaining the fidelity of a homogenized description. To the authors' knowledge, this is the first study where the effective boundary conditions in quasi-periodic media have been considered.

\section{Problem statement}

Assuming all parameters and variables hereon to be dimensionless (with reference to a suitable dimensional platform), we consider the scalar wave motion in an unbounded heterogeneous medium, namely

$$
\nabla \cdot(G(\boldsymbol{x}) \nabla u)+\rho(\boldsymbol{x}) \omega^{2} u=0, \quad \boldsymbol{x} \in \mathbb{R}^{d},
$$

where $d \geqslant 1$ and $\omega$ denotes the oscillation frequency. With reference to Fig. 1, we let the propagation medium feature both smooth (but otherwise arbitrary) macroscopic variation, and periodic small-scale fluctuation; specifically, we assume the coefficients $G(\boldsymbol{x})>0$ and $\rho(\boldsymbol{x})>0$ in (1) to admit either additive or multiplicative separation between "macroscopic" and "microscopic" variations according to

$$
\begin{aligned}
& \text { additive separation: } G(\boldsymbol{x})=G^{\prime}(\boldsymbol{x})+G^{\prime \prime}(\boldsymbol{x} / \epsilon), \quad \rho(\boldsymbol{x})=\rho^{\prime}(\boldsymbol{x})+\rho^{\prime \prime}(\boldsymbol{x} / \epsilon) \\
& \text { multiplicative separation: } \quad G(\boldsymbol{x})=G^{\prime}(\boldsymbol{x}) G^{\prime \prime}(\boldsymbol{x} / \epsilon), \quad \rho(\boldsymbol{x})=\rho^{\prime}(\boldsymbol{x}) \rho^{\prime \prime}(\boldsymbol{x} / \epsilon)
\end{aligned}
$$

where $\epsilon=o(1)$ is the germane perturbation parameter; $G^{\prime}>0$ and $\rho^{\prime}>0$ are bounded and smooth functions supported in $\mathbb{R}^{d}$; and $G^{\prime \prime}$ and $\rho^{\prime \prime}$ are $Y$-periodic functions, $Y \subset \mathbb{R}^{d}$. Making reference to a Cartesian coordinate system tied to an orthonormal basis $\boldsymbol{e}_{j}(j=\overline{1, d})$, we define the unit cell of periodicity as

$$
Y=\left\{\boldsymbol{x}: 0<\boldsymbol{x} \cdot \boldsymbol{e}_{j}<\ell_{j}, j=\overline{1, d}\right\}, \quad|Y|=1
$$

Remark 1. In the context of linear elasticity and anti-plane shear waves, $u, G$ and $\rho$ in (1) can be interpreted (for $d \in\{1,2\}$ ) as the transverse displacement, shear modulus, and mass density, respectively.

Remark 2. In situations where $G^{\prime \prime}(\boldsymbol{x} / \epsilon)$ and $\rho^{\prime \prime}(\boldsymbol{x} / \epsilon)$ are discontinuous, (1) is implicitly complemented by the "perfect bonding" conditions between smooth (microscopic) constituents. Letting $\Gamma_{\epsilon} \subset \mathbb{R}^{d-1}$ denote the 
union of all such material discontinuities in $\mathbb{R}^{d}$, we hereon implicitly assume that

$$
\llbracket u \rrbracket=0, \quad \llbracket \boldsymbol{n} \cdot(G \nabla u) \rrbracket=0, \quad \boldsymbol{x} \in \Gamma_{\epsilon}
$$

where $\boldsymbol{n}$ denotes the unit normal on $\Gamma_{\epsilon}$, and

$$
\llbracket g \rrbracket(\boldsymbol{x})=\lim _{\eta \rightarrow 0^{+}}\{g(\boldsymbol{x}+\eta \boldsymbol{n}(\boldsymbol{x}))-g(\boldsymbol{x}-\eta \boldsymbol{n}(\boldsymbol{x}))\}, \quad \boldsymbol{x} \in \Gamma_{\epsilon}
$$

signifies the jump across the interface.

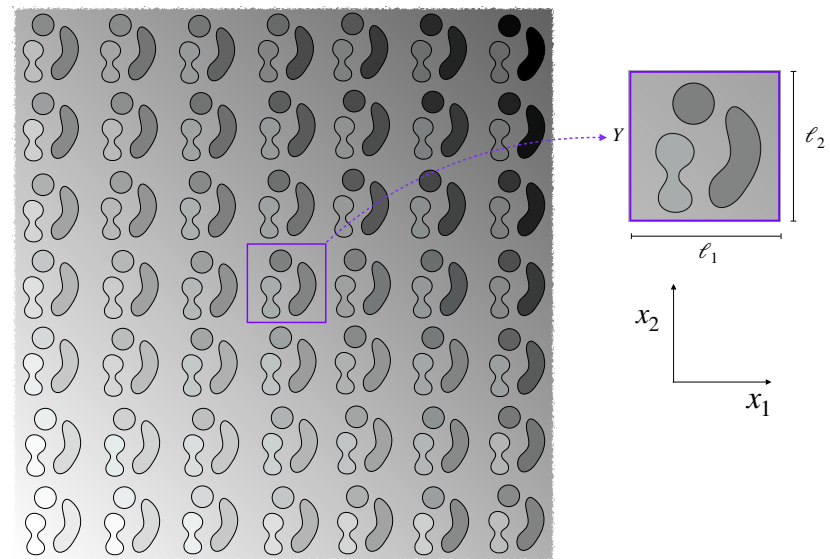

Figure 1: Quasi-periodic medium featuring (i) smooth macroscopic variation, and (ii) periodic (but not necessarily smooth) microscopic fluctuation.

\subsection{Objective and assumptions}

At this point, we observe from the representation (2)-(3) of $G(\boldsymbol{x})$ and $\rho(\boldsymbol{x})$ that the "microscopic" coefficient fluctuations in (1) are in fact $\epsilon Y$-periodic. In this setting, our goal is to obtain an effective i.e. macroscopic representation of (1), given by a field equation (with smoothly varying coefficients) governing the "mean" wave motion, assuming that:

- the frequency of oscillation is finite, namely $\omega=O(1)$, yet sufficiently low so that the germane wave dispersion - as driven by the "macro" and "micro" medium heterogeneities - resides inside the (apparent) first pass band [12]; and

- the characteristic lengthscale of $\epsilon Y$ is much smaller than the $\omega$-induced dominant wavelength of $u(\boldsymbol{x})$, as implied by the premise $\epsilon=o(1)$.

Motivated by the earlier studies of waves in periodic media [11, 27, 25, 7, 4, we tackle the problem via multiple-scales asymptotic expansion [5, 9, that revolves around the mapping $u(\boldsymbol{x}) \mapsto u(\boldsymbol{x}, \boldsymbol{x} / \epsilon)$ as a means to 
parse the macroscopic and microscopic wavefield fluctuations. In this setting, we also note that the foregoing restriction on $\omega$ amounts to considering the low-frequency, low-wavenumber (LF-LW) homogenization 35, 31] of wave motion. For completeness we pursue the asymptotic approximation up to the second order, which brings about an effective field equation that captures with high fidelity the combined effects of macroscopic and microscopic medium heterogeneities.

Remark 3. In periodic media, the sought second-order correction - appearing as a singular perturbation of the leading-order effective field equation [4, 46] - has only a moderate effect [46] on the (frequency-dependent) phase of the solution. However, a recent study [19] demonstrates that such correction may have a major effect of the solution amplitude when considering wave motion in bounded micro-structured domains.

In what follows, we pursue the LF-LW asymptotic treatment i.e. homogenization of (1), assuming either (2) or 3 , in terms of the perturbation parameter $\epsilon$. For brevity of presentation, we describe in detail the homogenization of one-dimensional (1D) waves, and follow up by presenting only the final result for the general case in $\mathbb{R}^{d}, d \geqslant 1$ - obtained in an analogous fashion. We then complete the $1 \mathrm{D}$ analysis by obtaining (also via multiple scales expansion) the effective boundary conditions applicable to the mean-field motion, and we illustrate numerically the analytical developments by considering $1 \mathrm{D}$ waves in both unbounded and bounded quasi-periodic domains.

\section{Effective field equation for one-dimensional problems}

When $d=1$, field equation (1) reduces to

$$
\frac{\mathrm{d}}{\mathrm{d} x}\left(G(x) \frac{\mathrm{d} u}{\mathrm{~d} x}\right)+\rho(x) \omega^{2} u=0, \quad x \in \mathbb{R} .
$$

Here $G(x)$ and $\rho(x)$ satisfy the one-dimensional counterparts of either (2) or (3), where $G^{\prime \prime}(x / \epsilon)$ and $\rho^{\prime \prime}(x / \epsilon)$ are $\epsilon Y$-periodic with $Y=(0,1)$. Within the framework of two-scale homogenization [9], this motivates introduction of the "fast" coordinate $y=\epsilon^{-1} x$ and affiliated mappings

$$
\begin{array}{rlrl}
u(x) & \mapsto u(x, y), & \frac{\mathrm{d}}{\mathrm{d} x} & \mapsto \frac{\partial}{\partial x}+\epsilon^{-1} \frac{\partial}{\partial y}, \\
G(x) & \mapsto G(x, y), \quad \rho(x) \mapsto \rho(x, y),
\end{array}
$$

designed to separate the macroscopic variations (described in terms of $x$ ) from their microscopic counterparts (by definition $Y$-periodic in terms of $y$ ). In terms of $(2)-(3)$, we specifically see that

$$
\begin{array}{rr}
\text { additive separation: } & G(x, y)=G^{\prime}(x)+G^{\prime \prime}(y), \quad \rho(x, y)=\rho^{\prime}(x)+\rho^{\prime \prime}(y) \\
\text { multiplicative separation: } & G(x, y)=G^{\prime}(x) G^{\prime \prime}(y), \quad \rho(x, y)=\rho^{\prime}(x) \rho^{\prime \prime}(y)
\end{array}
$$

For convenience, we also introduce a two-scale flux quantity $\sigma$ - namely the shear stress in terms of anti-plane shear waves - and its mapping via

$$
\sigma(x):=G(x) \frac{\mathrm{d} u}{\mathrm{~d} x} \quad \mapsto \quad \sigma(x, y)=G(x, y)\left(\frac{\partial u}{\partial x}+\epsilon^{-1} \frac{\partial u}{\partial y}\right) .
$$


On substituting (7) into (6) and using short-hand notation $(\cdot)_{, x}=\partial(\cdot) / \partial x$ and $(\cdot)_{, y}=\partial(\cdot) / \partial y$, (6) can be rewritten in powers of $\epsilon$ as

$$
\epsilon^{-2}\left(\left[G u_{, y}\right]_{, y}\right)+\epsilon^{-1}\left(\left[G u_{, y}\right]_{, x}+\left[G u_{, x}\right]_{, y}\right)+\left(\rho \omega^{2} u+\left[G u_{, x}\right]_{, x}\right)=0, \quad x \in \mathbb{R}, y \in Y .
$$

We next pursue the asymptotic solution of (11) in terms of the ansatz

$$
u(x, y)=\sum_{k=0}^{\infty} \epsilon^{k} u_{k}(x, y), \quad \sigma(x, y)=\sum_{k=0}^{\infty} \epsilon^{k} \sigma_{k}(x, y)
$$

where $\sigma_{k}=G\left(u_{k, x}+u_{k+1, y}\right)$. With such definitions, the homogenized i.e. macroscopic LF-LW description of the problem can be effected in terms of the "mean" wave motion

$$
\langle u\rangle(x):=\int_{0}^{1} u(x, y) \mathrm{d} y=\sum_{k=0}^{\infty} \epsilon^{k}\left\langle u_{k}\right\rangle(x) .
$$

In the sequel, we shall also make use of the partial sums

$$
u^{[p]}(x, y)=\sum_{k=0}^{p} \epsilon^{k} u_{k}(x, y), \quad \sigma^{[p]}(x, y)=\sum_{k=0}^{p} \epsilon^{k} \sigma_{k}(x, y), \quad\langle u\rangle^{[p]}(x)=\sum_{k=0}^{p} \epsilon^{k}\left\langle u_{k}\right\rangle(x) .
$$

\subsection{O(1) homogenization}

By virtue of 12 , the $O\left(\epsilon^{-2}\right)$ statement of 11 becomes

$$
\left[G u_{0, y}\right]_{, y}=0 \quad \Rightarrow \quad u_{0, y}=G^{-1} c(x), \quad y \in Y
$$

where $x \in \mathbb{R}$ hereon, and $c(x)$ is a constant of integration. On averaging the last result, we obtain

$$
\left\langle u_{0, y}\right\rangle=c(x)\left\langle G^{-1}\right\rangle
$$

Thanks to the $Y$-periodicity of $u_{0}(\cdot, y)$, the left-hand side of (17) vanishes identically whereby $c(x)=0$. Accordingly, the leading-order solution depends exclusively on the macroscopic variable and we write

$$
u_{0}(x, y)=\left\langle u_{0}\right\rangle
$$

On collecting the $O\left(\epsilon^{-1}\right)$ terms in (11), one arrives at

$$
\left[G\left(u_{1, y}+u_{0, x}\right)\right]_{, y}=0, \quad y \in Y
$$

Since 18 is a linear ordinary differential equation in $y$, its general solution can be written as

$$
u_{1}(x, y)=\left\langle u_{1}\right\rangle+P(x, y)\left\langle u_{0}\right\rangle_{, x}, \quad\langle P\rangle=0,
$$

where $P$ is a zero-mean ( $Y$-periodic in $y$ ) cell function that satisfies

$$
\left[G\left(1+P_{, y}\right)\right]_{, y}=0, \quad y \in Y
$$


for each $x \in \mathbb{R}$. Next, we proceed to the $O(1)$ governing equation which reads

$$
\left[G\left(u_{2, y}+u_{1, x}\right)\right]_{, y}+\left[G\left(u_{1, y}+u_{0, x}\right)\right]_{, x}+\rho \omega^{2}\left\langle u_{0}\right\rangle=0, \quad y \in Y
$$

Again, 21 is a linear equation whereby its solution admits the representation

$$
u_{2}(x, y)=\left\langle u_{2}\right\rangle+P(x, y)\left\langle u_{1}\right\rangle_{, x}+\tilde{P}(x, y)\left\langle u_{0}\right\rangle_{, x}+Q(x, y)\left\langle u_{0}\right\rangle_{, x x}, \quad\langle\tilde{P}\rangle=\langle Q\rangle=0
$$

where $\tilde{P}$ and $Q$ are zero-mean cell functions satisfying

$$
\left(\left[G\left(1+P_{, y}\right)\right]_{, x}+\left[G\left(P_{, x}+\tilde{P}_{, y}\right)\right]_{, y}\right)\left\langle u_{0}\right\rangle_{, x}+\left(G\left(1+P_{, y}\right)+\left[G\left(P+Q_{, y}\right)\right]_{, y}\right)\left\langle u_{0}\right\rangle_{, x x}+\rho \omega^{2}\left\langle u_{0}\right\rangle=0, \quad y \in Y
$$

thanks to 19 .

Remark 4. Up to this point, the key structural difference between the present problem and that describing the wave motion in periodic media [46] is the presence of the cell function $\tilde{P}(x, y)$ in the expression [22] for $u_{2}$. As will be shown in the sequel, this function vanishes identically when $G^{\prime}(x)$ and $\rho^{\prime}(x)$ in (8)-(9) are constant.

Integrating 21) over $Y$, taking into account the inherent $Y$-periodicity in the fast variable, and assuming $P, Q$ and $\tilde{P}$ to be bounded, we obtain the (leading-order) homogenized field equation governing $\left\langle u_{0}\right\rangle$, namely

$$
\left[\mu^{(0)}(x)\left\langle u_{0}\right\rangle_{, x}\right]_{, x}+\varrho^{(0)}(x) \omega^{2}\left\langle u_{0}\right\rangle=0, \quad x \in \mathbb{R}
$$

whose macroscopically-heterogeneous effective coefficients are given by

$$
\mu^{(0)}(x)=\left\langle G\left(1+P_{, y}\right)\right\rangle, \quad \varrho^{(0)}(x)=\langle\rho\rangle .
$$

In contrast to the purely periodic case [24, 46], we note that even the leading-order effective model is in this case dispersive due to macroscopic variation (25) of the effective medium properties. We also note that exposing the (heterogeneous) effective medium properties requires knowledge of the cell function $P(x, y)$ whose evaluation, and that of its companions, is addressed in Section 3.5

\section{2. $O(\epsilon)$ homogenization}

On collecting the $O(\epsilon)$ terms in (11), we find

$$
\left[G\left(u_{3, y}+u_{2, x}\right)\right]_{, y}+\left[G\left(u_{2, y}+u_{1, x}\right)\right]_{, x}+\rho \omega^{2} u_{1}=0, \quad y \in Y .
$$

By analogy to earlier treatment, the linearity of 26) allows us to write the general solution as

$$
\begin{aligned}
& u_{3}(x, y)=\left\langle u_{3}\right\rangle+P(x, y)\left\langle u_{2}\right\rangle_{, x}+\tilde{P}(x, y)\left\langle u_{1}\right\rangle_{, x}+Q(x, y)\left\langle u_{1}\right\rangle_{, x x}+ \\
& \tilde{R}(x, y)\left\langle u_{0}\right\rangle_{, x}+\tilde{Q}(x, y)\left\langle u_{0}\right\rangle_{, x x}+R(x, y)\left\langle u_{0}\right\rangle_{, x x x}, \quad\langle\tilde{Q}\rangle=\langle R\rangle=\langle\tilde{R}\rangle=0,
\end{aligned}
$$


where the zero-mean cell functions $\tilde{Q}, R$ and $\tilde{R}$ are such that

$$
\begin{array}{r}
\left(\left[G\left(1+P_{, y}\right)\right]_{, x}+\left[G\left(\tilde{P}_{, y}+P_{, x}\right)\right]_{, y}\right)\left\langle u_{1}\right\rangle_{, x}+\left(\left[G\left(Q_{, y}+P\right)\right]_{, y}+G\left(1+P_{, y}\right)\right)\left\langle u_{1}\right\rangle_{, x x}+ \\
\left(\left[G\left(P_{, x}+\tilde{P}_{, y}\right)\right]_{, x}+\left[G\left(\tilde{R}_{, y}+\tilde{P}_{, x}\right)\right]_{, y}+\rho \omega^{2} P\right)\left\langle u_{0}\right\rangle_{, x}+\left(\left[G\left(\tilde{Q}_{, y}+Q_{, x}+\tilde{P}\right)\right]_{, y}+\left[G\left(P+Q_{, y}\right)\right]_{, x}+G\left(P_{, x}+\tilde{P}_{, y}\right)\right)\left\langle u_{0}\right\rangle_{, x x} \\
+\left(\left[G\left(R_{, y}+Q\right)\right]_{, y}+G(P+Q, y)\right)\left\langle u_{0}\right\rangle_{, x x x}+\rho \omega^{2}\left\langle u_{1}\right\rangle=0, \quad(28)
\end{array}
$$

On integrating (28) over $Y$ and exploiting the periodicity of featured quantities, we obtain the effective field equation governing the first-order corrector $\left\langle u_{1}\right\rangle$ as

$$
\left[\mu^{(0)}(x)\left\langle u_{1}\right\rangle_{, x}\right]_{, x}+\varrho^{(0)}(x) \omega^{2}\left\langle u_{1}\right\rangle=-\left[\eta_{, x}(x)+\varrho^{(1)}(x) \omega^{2}\right]\left\langle u_{0}\right\rangle_{, x}-\left[\eta(x)+\mu_{, x}^{(1)}(x)\right]\left\langle u_{0}\right\rangle_{, x x}-\mu^{(1)}(x)\left\langle u_{0}\right\rangle_{, x x x},
$$

whose "functionally-graded" coefficients are given by

$$
\mu^{(1)}(x)=\left\langle G\left(P+Q_{, y}\right)\right\rangle, \quad \varrho^{(1)}(x)=\langle\rho P\rangle, \quad \eta(x)=\left\langle G\left(P_{, x}+\tilde{P}_{, y}\right)\right\rangle .
$$

\section{3. $O\left(\epsilon^{2}\right)$ homogenization}

On collecting the $O\left(\epsilon^{2}\right)$ terms in 11 , one finds that

$$
\left[G\left(u_{4, y}+u_{3, x}\right)\right]_{, y}+\left[G\left(u_{3, y}+u_{2, x}\right)\right]_{, x}+\rho \omega^{2} u_{2}=0,
$$

Following the previously established analysis and integrating (31) over $Y$, we obtain the effective equation for $\left\langle u_{2}\right\rangle$, namely

$$
\begin{aligned}
& {\left[\mu^{(0)}(x)\left\langle u_{2}\right\rangle_{, x}\right]_{, x}+\varrho^{(0)}(x) \omega^{2}\left\langle u_{2}\right\rangle=-\left[\eta_{, x}(x)+\varrho^{(1)}(x) \omega^{2}\right]\left\langle u_{1}\right\rangle_{, x}-\left[\eta(x)+\mu_{, x}^{(1)}(x)\right]\left\langle u_{1}\right\rangle_{, x x}-\mu^{(1)}(x)\left\langle u_{1}\right\rangle_{, x x x}} \\
& -\left[\phi_{, x}(x)+\tilde{\varrho}^{(2)}(x) \omega^{2}\right]\left\langle u_{0}\right\rangle_{, x}-\left[\psi_{, x}(x)+\phi(x)+\varrho^{(2)}(x) \omega^{2}\right]\left\langle u_{0}\right\rangle_{, x x}-\left[\psi(x)+\mu_{, x}^{(2)}(x)\right]\left\langle u_{0}\right\rangle_{, x x x}-\mu^{(2)}(x)\left\langle u_{0}\right\rangle_{, x x x x}
\end{aligned}
$$

where

$$
\begin{array}{ll}
\mu^{(2)}(x)=\left\langle G\left(Q+R_{, y}\right)\right\rangle, & \varrho^{(2)}(x)=\langle\rho Q\rangle, \quad \tilde{\varrho}^{(2)}(x)=\langle\rho \tilde{P}\rangle, \\
\phi(x)=\left\langle G\left(\tilde{P}_{, x}+\tilde{R}_{, y}\right)\right\rangle, \quad \psi(x)=\left\langle G\left(Q_{, x}+\tilde{P}+\tilde{Q}_{, y}\right)\right\rangle .
\end{array}
$$

\subsection{Macroscopic description of the mean wave motion}

By virtue of 13, one my conveniently compute the weighted sum $(24)+\epsilon(29)+\epsilon^{2}(32)$, resulting in the second-order effective equation

$$
\begin{aligned}
& \mu^{(0)}(x)\langle u\rangle_{, x x}+\mu_{, x}^{(0)}(x)\langle u\rangle_{, x}+\varrho^{(0)}(x) \omega^{2}\langle u\rangle= \\
& -\epsilon\left(\left[\eta_{, x}(x)+\varrho^{(1)}(x) \omega^{2}\right]\langle u\rangle_{, x}+\left[\eta(x)+\mu_{, x}^{(1)}(x)\right]\langle u\rangle_{, x x}+\mu^{(1)}(x)\langle u\rangle_{, x x x}\right) \\
& -\epsilon^{2}\left(\left[\phi_{, x}(x)+\varrho^{(2)}(x) \omega^{2}\right]\langle u\rangle_{, x}+\left[\psi_{, x}(x)+\phi(x)+\varrho^{(2)}(x) \omega^{2}\right]\langle u\rangle_{, x x}\right. \\
& \left.+\left[\psi(x)+\mu_{, x}^{(2)}(x)\right]\langle u\rangle_{, x x x}+\mu^{(2)}(x)\langle u\rangle_{, x x x x}\right)+O\left(\epsilon^{3}\right),
\end{aligned}
$$


which demonstrates that an $O(\epsilon)$ (resp. $O\left(\epsilon^{2}\right)$ ) correction of the leading-order effective model

$$
\left[\mu^{(0)}(x)\langle u\rangle_{, x}\right]_{, x}+\varrho^{(0)}(x) \omega^{2}\langle u\rangle=0
$$

due to presence of two-scale medium variations entails spatial derivatives up to order three (resp. four). One key difference between the current model and its counterparts for periodic media [24, 46] where $G(x, y)=G(y)$ and $\rho(x, y)=\rho(y)$, however, is that (34) features a nontrivial $O(\epsilon)$ correction that can be shown to vanish identically in the periodic case [31. With reference to (8), for instance, it can be specifically shown that

- $G^{\prime}(x)=\rho^{\prime}(x)=$ const. (no macro-scale heterogeneities)

$$
\Longrightarrow \quad \mu^{(k)}=\text { const., } \quad \rho^{(k)}=\text { const. }(k=0,2), \quad \mu^{(1)}=\varrho^{(1)}=\tilde{\varrho}^{(2)}=\eta=\phi=\psi=0 ;
$$

- $G^{\prime \prime}(y)=\rho^{\prime \prime}(y)=0$ (no micro-scale heterogeneities)

$$
\Longrightarrow \quad \mu^{(0)}(x)=G^{\prime}(x), \quad \varrho^{(0)}(x)=\rho^{\prime}(x), \quad \mu^{(k)}=\rho^{(k)}=\tilde{\varrho}^{(2)}=\eta=\phi=\psi=0 ; \quad(k \geqslant 1) .
$$

For completeness, we also recall that the effective coefficients $\varrho^{(k)}, \mu^{(k)}(k=0,1, \ldots), \eta, \phi$, and $\psi$ are specified via (25), (30) and (33) in terms of the respective cell functions whose evaluation is examined next.

Remark 5. In the context of apparent complexity characterizing (34), a fair question to ask concerns the utility of homogenized descriptions for this class of problems: what is to be gained? Assuming multi-dimensional problems (a subject that will be addressed shortly), a short answer is "the computational efficiency" for such homogenized models are characterized by smooth coefficient variations. This is in contrast to the original quasi-periodic medium that may feature material discontinuities at the microscopic $(O(\epsilon))$ scale, and thus necessitate spatial discretization whose "fine" lengthscale is o $(\epsilon)$, i.e. several decades smaller than the dominant wavelength.

\subsection{Cell functions}

For generality, we assume (as examined earlier) that the scaled unit cell of periodicity $Y=(0,1)$ is composed of $N$ smoothly heterogeneous pieces i.e. sub-cells $Y_{q}, q=\overline{1, N}$ such that $G^{\prime \prime}(y)$ and $\rho^{\prime \prime}(y)$ are differentiable within each (open) set $Y_{q}$. In this setting, by recalling 20 we find that the cell function $P(x, y)$ featured in 19 solves the boundary value problem

$$
\begin{aligned}
& {\left[G\left(1+P_{, y}\right)\right]_{, y}=0, \quad y \in Y_{q}} \\
& P, G\left(1+P_{, y}\right) \quad Y \text {-periodic; }\langle P\rangle=0 \\
& \llbracket P \rrbracket=0, \quad \llbracket G\left(1+P_{, y}\right) \rrbracket=0, \quad y \in \partial Y_{q} \backslash \partial Y
\end{aligned}
$$

for any given $x \in \mathbb{R}$, where the term $G\left(1+P_{, y}\right)$ can be understood as a generalized flux relevant to $P$.

From the leading-order field equation (24), $\left\langle u_{0}\right\rangle$ can be expressed as a linear combination of $\left\langle u_{0}\right\rangle_{, x}$ and $\left\langle u_{0}\right\rangle_{, x x}$. Since $\left\langle u_{0}\right\rangle$ is a generic mean wavefield propagating through a heterogeneous medium, however, $\left\langle u_{0}\right\rangle_{, x}$ and $\left\langle u_{0}\right\rangle_{, x x}$ are themselves linearly independent. As a result, their respective multipliers in 23) must vanish independently 
for each $x$. By setting the multiplier therein of $\left\langle u_{0}\right\rangle_{, x x}$ to zero, we obtain the boundary value problem for $Q$ as

$$
\begin{aligned}
& {\left[G\left(P+Q_{, y}\right)\right]_{, y}=\frac{\rho}{\varrho^{(0)}} \mu^{(0)}-G(1+P, y), \quad y \in Y_{q}} \\
& Q, G\left(P+Q_{, y}\right) \quad Y \text {-periodic; } \quad\langle Q\rangle=0 \\
& \llbracket Q \rrbracket=0, \quad \llbracket G(P+Q, y) \rrbracket=0, \quad y \in \partial Y_{q} \backslash \partial Y
\end{aligned}
$$

that holds for each $x \in \mathbb{R}$. In this vein, the boundary value problem governing $\tilde{P}$ can be further identified as

$$
\begin{aligned}
& {\left[G\left(P_{, x}+\tilde{P}_{, y}\right)\right]_{, y}=\frac{\rho}{\varrho^{(0)}} \mu_{, x}^{(0)}-\left[G\left(1+P_{, y}\right)\right]_{, x}, \quad y \in Y_{q}} \\
& \tilde{P}, G\left(P_{, x}+\tilde{P}_{, y}\right) \quad Y \text {-periodic; } \quad\langle\tilde{P}\rangle=0 \\
& \llbracket \tilde{P} \rrbracket=0, \quad \llbracket G\left(P_{, x}+\tilde{P}_{, y}\right) \rrbracket=0, \quad y \in \partial Y_{q} \backslash \partial Y .
\end{aligned}
$$

Proceeding with the analysis, we similarly find from $(28)$ the respective boundary value problems governing $\tilde{Q}, R$ and $\tilde{R}$ to read

$$
\begin{aligned}
& {\left[G\left(\tilde{P}+Q_{, x}+\tilde{Q}_{, y}\right)\right]_{, y}=\frac{\rho}{\varrho^{(0)}}\left(\eta+\mu_{, x}^{(1)}\right)-} \\
& \quad\left[G\left(P+Q_{, y}\right)\right]_{, x}-G\left(P_{, x}+\tilde{P}_{, y}\right) \\
& \left.+\rho-\frac{\varrho^{(1)}}{\varrho^{(0)}}\right)\left(\frac{\mu_{, x}^{(0)}}{\varrho^{(0)}}+\left[\frac{\mu^{0}}{\varrho^{(0)}}\right]_{, x}\right), \quad y \in Y_{q} \\
& \tilde{Q}, G\left(\tilde{P}+Q_{, x}+\tilde{Q}_{, y}\right), \quad Y \text {-periodic; } \quad\langle\tilde{Q}\rangle=0 \\
& \llbracket \tilde{Q} \rrbracket=0, \quad \llbracket G\left(\tilde{P}+Q_{, x}+\tilde{Q}_{, y}\right) \rrbracket=0, \quad y \in \partial Y_{q} \backslash \partial Y, \\
& {\left[G\left(Q+R_{, y}\right)\right]_{, y}=\frac{\rho}{\varrho^{(0)}} \mu^{(1)}-G\left(P+Q_{, y}\right)+\rho \frac{\mu^{(0)}}{\varrho^{(0)}}\left(P-\frac{\varrho^{(1)}}{\varrho^{(0)}}\right), \quad y \in Y_{q}} \\
& R, G\left(Q+R, R_{, y}\right), \quad Y \text {-periodic; } \quad\langle R\rangle=0 \\
& \llbracket R \rrbracket=0, \quad \llbracket G(Q+R, y) \rrbracket=0, \quad y \in \partial Y_{q} \backslash \partial Y,
\end{aligned}
$$

and

$$
\begin{aligned}
& {\left[G\left(\tilde{R}_{, y}+\tilde{P}_{, x}\right)\right]_{, y}=\frac{\rho}{\varrho^{(0)}} \eta_{, x}-\left[G\left(P_{, x}+\tilde{P}_{, y}\right)\right]_{, x}+\rho\left(P-\frac{\varrho^{(1)}}{\varrho^{(0)}}\right)\left[\frac{\mu_{, x}^{(0)}}{\varrho^{(0)}}\right]_{, x}, \quad y \in Y_{q}} \\
& \tilde{R}, \quad G\left(\tilde{R}_{, y}+\tilde{P}_{, x}\right), \quad Y \text {-periodic; } \quad\langle\tilde{R}\rangle=0 \\
& \llbracket \tilde{R} \rrbracket=0, \quad \llbracket G\left(\tilde{R}_{, y}+\tilde{P}_{, x}\right) \rrbracket=0, \quad y \in \partial Y_{q} \backslash \partial Y,
\end{aligned}
$$

for any given $x \in \mathbb{R}$.

Remark 6. Thanks to the results in [9] (Chapter 2), one finds that the boundary value problems [35]-40] are well-posed, each featuring a field equation with the common principal part $\left[G(\cdot)_{, y}\right]_{, y}$. Therein, the dependence on the macroscopic variable $x$ is injected via smooth coefficient variations $G^{\prime}(x)$ and $\rho^{\prime}(x)$ according to either (8) or (9). As a result, the cell functions $\{P, Q, R\}$ and $\{\tilde{P}, \tilde{Q}, \tilde{R}\}$ are likewise smooth functions of $x$, resulting in the smooth spatial variation of the effective coefficients $\mu^{(k)}, \varrho^{(k)}(k=0,1, \ldots), \varrho^{(2)}, \eta, \phi$, and $\psi$. In the context of numerical (e.g. finite element) implementation, such "functionally-graded" coefficients can then be sampled with suitable density in $x$ and interpolated accordingly. In other words, even though the cell problems (35)-(40) are dependent on the macroscopic variable $x$, their solution would need to be sampled only over a relatively coarse grid. 


\subsection{Cell stresses and stress expansion}

With the cell functions at hand, we next introduce the so-called cell stresses $\Sigma_{j}(j=\overline{0,5})$ as

$$
\begin{array}{llrl}
\Sigma_{0}(x, y) & =\frac{G\left(1+P_{, y}\right)}{\mu^{(0)}}, & \Sigma_{1}(x, y) & =\frac{G\left(P+Q_{, y}\right)}{\mu^{(0)}}, \\
\Sigma_{2}(x, y) & =\frac{G\left(P_{, x}+\tilde{P}_{, y}\right)}{\mu^{(0)}}, & \Sigma_{3}(x, y) & =\frac{G\left(\tilde{R}_{, y}+\tilde{P}_{, x}\right)}{\mu^{(0)}}, \\
\Sigma_{4}(x, y) & =\frac{G\left(\tilde{P}+Q_{, x}+\tilde{Q}_{, y}\right)}{\mu^{(0)}}, & \Sigma_{5}(x, y) & =\frac{G\left(Q+R_{, y}\right)}{\mu^{(0)}},
\end{array}
$$

By way of 25) and (35), one can show that $\Sigma_{0}(x, y)=1$; for clarity of discussion, however, we will retain this term "as is" wherever it appears. Thanks to (7), (12) and (41), the stress field (10) affiliated with $u(x, y)$ can be expanded as

$$
\begin{aligned}
\sigma(x, y)=\mu^{(0)}(x)\left[\left(\Sigma_{0}(x, y)+\epsilon \Sigma_{2}(x, y)\right.\right. & \left.+\epsilon^{2} \Sigma_{3}(x, y)\right)\langle u\rangle_{, x}^{[2]} \\
+ & \left.\left(\epsilon \Sigma_{1}(x, y)+\epsilon^{2} \Sigma_{4}(x, y)\right)\langle u\rangle_{, x x}^{[2]}+\epsilon^{2} \Sigma_{5}(x, y)\langle u\rangle_{, x x x}^{[2]}\right]+O\left(\epsilon^{3}\right) .
\end{aligned}
$$

\section{Effective field equation in $\mathbb{R}^{d}(d>1)$}

In this section, we apply the foregoing two-scale analysis to the original problem (1) in $\mathbb{R}^{d}$. For brevity, we show only the essential definitions and homogenization results. We start by introducing the "fast" spatial coordinate $\boldsymbol{y}=\epsilon^{-1} \boldsymbol{x}$ and mappings

$$
\begin{array}{ll}
u(\boldsymbol{x}) \Rightarrow u(\boldsymbol{x}, \boldsymbol{y}), & G(\boldsymbol{x}) \Rightarrow G(\boldsymbol{x}, \boldsymbol{y}), \\
\rho(\boldsymbol{x}) \Rightarrow \rho(\boldsymbol{x}, \boldsymbol{y}), & \nabla \Rightarrow \nabla_{\boldsymbol{x}}+\frac{1}{\epsilon} \nabla_{\boldsymbol{y}},
\end{array}
$$

which transforms (1) into

$$
\epsilon^{-2}\left[\nabla_{\boldsymbol{y}} \cdot\left(G \nabla_{\boldsymbol{y}} u\right)\right]+\epsilon^{-1}\left[\nabla_{\boldsymbol{x}} \cdot\left(G \nabla_{\boldsymbol{y}} u\right)+\nabla_{\boldsymbol{y}} \cdot\left(G \nabla_{\boldsymbol{x}} u\right)\right]+\left[\rho \omega^{2} u+\nabla_{\boldsymbol{x}} \cdot\left(G \nabla_{\boldsymbol{x}} u\right)\right]=0 .
$$

We then pursue the ansatz

$$
u(\boldsymbol{x}, \boldsymbol{y})=\sum_{k=0}^{\infty} \epsilon^{k} u_{k}(\boldsymbol{x}, \boldsymbol{y}) \quad \Longrightarrow \quad\langle u\rangle(\boldsymbol{x})=\int_{Y} u(\boldsymbol{x}, \boldsymbol{y}) \mathrm{d} \boldsymbol{y}=\sum_{k=0}^{\infty} \epsilon^{k}\left\langle u_{k}\right\rangle(\boldsymbol{x}) .
$$

By retracing the steps of one-dimensional analysis, we specifically find that

$$
u_{2}(\boldsymbol{x}, \boldsymbol{y})=\left\langle u_{2}\right\rangle+\boldsymbol{P}(\boldsymbol{x}, \boldsymbol{y}) \cdot \nabla\left\langle u_{1}\right\rangle+\boldsymbol{Q}(\boldsymbol{x}, \boldsymbol{y}): \nabla \nabla\left\langle u_{0}\right\rangle+\tilde{\boldsymbol{P}}(\boldsymbol{x}, \boldsymbol{y}) \cdot \nabla\left\langle u_{0}\right\rangle, \quad\langle\boldsymbol{Q}\rangle=\langle\boldsymbol{P}\rangle=\langle\tilde{\boldsymbol{P}}\rangle=\mathbf{0},
$$

where $\boldsymbol{P}, \boldsymbol{Q}$ and $\tilde{\boldsymbol{P}}$ are tensorial cell functions reading

$$
\boldsymbol{P}=P_{i} \boldsymbol{e}_{i}, \quad \boldsymbol{Q}=Q_{i j} \boldsymbol{e}_{i} \otimes \boldsymbol{e}_{j}, \quad \tilde{\boldsymbol{P}}=\tilde{P}_{i} \boldsymbol{e}_{i}
$$

in dyadic notation, which assumes implicit summation over repeated indexes $i, j=\overline{1, d}$. In 46] and thereafter, we use symbol ":" to indicate $n$-tuple contraction between two $n$th order tensors $(n \geqslant 2)$ producing a scalar. 
On further denoting by $\boldsymbol{I}_{n}$ the $n$th order symmetric identity tensor, the mean fields $\left\langle u_{k}\right\rangle(k=0,1,2)$ featured in 46 can be shown to satisfy the respective field equations

$$
\nabla \cdot\left[\boldsymbol{\mu}^{(0)}(\boldsymbol{x}) \cdot \nabla\left\langle u_{0}\right\rangle\right]+\varrho^{(0)}(\boldsymbol{x}) \omega^{2}\left\langle u_{0}\right\rangle=0
$$

where

$$
\begin{gathered}
\boldsymbol{\mu}^{(0)}(\boldsymbol{x})=\left\langle G\left(\boldsymbol{I}_{2}+\nabla_{\boldsymbol{y}} \boldsymbol{P}\right)\right\rangle, \quad \varrho^{(0)}(\boldsymbol{x})=\langle\rho\rangle ; \\
\nabla \cdot\left[\boldsymbol{\mu}^{(0)}(\boldsymbol{x}) \cdot \nabla\left\langle u_{1}\right\rangle\right]+\varrho^{(0)}(\boldsymbol{x}) \omega^{2}\left\langle u_{1}\right\rangle=-\left[\nabla \cdot \boldsymbol{\eta}(\boldsymbol{x})+\varrho^{(1)}(\boldsymbol{x}) \omega^{2}\right] \cdot \nabla\left\langle u_{0}\right\rangle \\
-\left[\nabla \cdot \boldsymbol{\mu}^{(1)}(\boldsymbol{x})+\boldsymbol{\eta}(\boldsymbol{x})\right]: \nabla \nabla\left\langle u_{0}\right\rangle-\boldsymbol{\mu}^{(1)}(\boldsymbol{x}): \nabla \nabla \nabla\left\langle u_{0}\right\rangle,
\end{gathered}
$$

where

$$
\boldsymbol{\mu}^{(1)}(\boldsymbol{x})=\left\langle G\left(\boldsymbol{I}_{2} \otimes \boldsymbol{P}+\nabla_{\boldsymbol{y}} \boldsymbol{Q}\right)\right\rangle, \quad \boldsymbol{\varrho}^{(1)}(\boldsymbol{x})=\langle\rho \boldsymbol{P}\rangle, \quad \boldsymbol{\eta}(\boldsymbol{x})=\left\langle G\left(\nabla_{\boldsymbol{x}} \boldsymbol{P}+\nabla_{\boldsymbol{y}} \tilde{\boldsymbol{P}}\right)\right\rangle
$$

and

$$
\begin{array}{r}
\nabla \cdot\left[\boldsymbol{\mu}^{(0)}(\boldsymbol{x}) \cdot \nabla\left\langle u_{2}\right\rangle\right]+\varrho^{(0)}(\boldsymbol{x}) \omega^{2}\left\langle u_{2}\right\rangle=-\left[\nabla \cdot \boldsymbol{\eta}(\boldsymbol{x})+\varrho^{(1)}(\boldsymbol{x}) \omega^{2}\right] \cdot \nabla\left\langle u_{1}\right\rangle-\left[\boldsymbol{\eta}(\boldsymbol{x})+\nabla \cdot \boldsymbol{\mu}^{(1)}(\boldsymbol{x})\right]: \nabla \nabla\left\langle u_{1}\right\rangle \\
-\boldsymbol{\mu}^{(1)}(\boldsymbol{x}): \nabla \nabla \nabla\left\langle u_{1}\right\rangle-\left[\nabla \cdot \boldsymbol{\phi}(\boldsymbol{x})+\tilde{\boldsymbol{\varrho}}^{(2)}(\boldsymbol{x}) \omega^{2}\right] \cdot \nabla\left\langle u_{0}\right\rangle-\left[\nabla \cdot \boldsymbol{\psi}(\boldsymbol{x})+\boldsymbol{\phi}(\boldsymbol{x})+\boldsymbol{\varrho}^{(2)}(\boldsymbol{x}) \omega^{2}\right]: \nabla \nabla\left\langle u_{0}\right\rangle \\
-\left[\boldsymbol{\psi}(\boldsymbol{x})+\nabla \cdot \boldsymbol{\mu}^{(2)}(\boldsymbol{x})\right]: \nabla \nabla \nabla\left\langle u_{0}\right\rangle-\boldsymbol{\mu}^{(2)}(\boldsymbol{x}): \nabla \nabla \nabla \nabla\left\langle u_{0}\right\rangle,
\end{array}
$$

where

$$
\begin{array}{ll}
\boldsymbol{\mu}^{(2)}(\boldsymbol{x})=\left\langle G\left(\boldsymbol{I}_{2} \otimes \boldsymbol{Q}+\nabla_{\boldsymbol{y}} \boldsymbol{R}\right)\right\rangle, \quad \boldsymbol{\varrho}^{(2)}(\boldsymbol{x})=\langle\rho \boldsymbol{Q}\rangle, & \tilde{\boldsymbol{\varrho}}^{(2)}(\boldsymbol{x})=\langle\rho \tilde{\boldsymbol{P}}\rangle, \\
\boldsymbol{\phi}(\boldsymbol{x})=\left\langle G\left(\nabla_{\boldsymbol{x}} \tilde{\boldsymbol{P}}+\nabla_{\boldsymbol{y}} \tilde{\boldsymbol{R}}\right)\right\rangle, \quad \boldsymbol{\psi}(\boldsymbol{x})=\left\langle G\left(\nabla_{\boldsymbol{x}} \boldsymbol{Q}+\boldsymbol{I}_{2} \otimes \tilde{\boldsymbol{P}}+\nabla_{\boldsymbol{y}} \tilde{\boldsymbol{Q}}\right)\right\rangle .
\end{array}
$$

From the weighted sum $447+\epsilon 49)+\epsilon^{2}(51)$, we obtain the effective field equation

$$
\begin{gathered}
\nabla \cdot\left[\boldsymbol{\mu}^{(0)}(\boldsymbol{x}) \cdot \nabla\langle u\rangle\right]+\varrho^{(0)}(\boldsymbol{x}) \omega^{2}\langle u\rangle= \\
-\epsilon\left(\left[\nabla \cdot \boldsymbol{\eta}(\boldsymbol{x})+\varrho^{(1)}(\boldsymbol{x}) \omega^{2}\right] \cdot \nabla\langle u\rangle+\left[\boldsymbol{\eta}(\boldsymbol{x})+\nabla \cdot \boldsymbol{\mu}^{(1)}(\boldsymbol{x})\right]: \nabla \nabla\langle u\rangle+\boldsymbol{\mu}^{(1)}(\boldsymbol{x}): \nabla \nabla \nabla\langle u\rangle\right) \\
-\epsilon^{2}\left(\left[\nabla \cdot \boldsymbol{\phi}(\boldsymbol{x})+\tilde{\boldsymbol{\varrho}}^{(2)}(\boldsymbol{x}) \omega^{2}\right] \cdot \nabla\langle u\rangle+\left[\nabla \cdot \boldsymbol{\psi}(\boldsymbol{x})+\boldsymbol{\phi}(\boldsymbol{x})+\boldsymbol{\varrho}^{(2)}(\boldsymbol{x}) \omega^{2}\right]: \nabla \nabla\langle u\rangle\right. \\
\left.\quad+\left[\boldsymbol{\psi}(\boldsymbol{x})+\nabla \cdot \boldsymbol{\mu}^{(2)}(\boldsymbol{x})\right]: \nabla \nabla \nabla\langle u\rangle+\boldsymbol{\mu}^{(2)}: \nabla \nabla \nabla \nabla\langle u\rangle\right)+O\left(\epsilon^{3}\right),
\end{gathered}
$$

where the cell functions needed to compute the effective coefficients $\boldsymbol{P} \in \mathbb{R}^{d}, \boldsymbol{Q} \in \mathbb{R}^{d \times d}, \boldsymbol{R} \in \mathbb{R}^{d \times d \times d}, \tilde{\boldsymbol{P}} \in$ $\mathbb{R}^{d}, \tilde{\boldsymbol{Q}} \in \mathbb{R}^{d \times d}$ and $\tilde{\boldsymbol{R}} \in \mathbb{R}^{d}$ according to 480 , (50) and (52) are given in Appendix A

Remark 7. Taking into account the above tensorial character of the respective cell functions, one finds from (48), 50) and 52 that $\varrho^{(0)}$ is a scalar; $\boldsymbol{\rho}^{(1)}$ and $\tilde{\boldsymbol{\rho}}^{(2)}$ are vectors; $\boldsymbol{\mu}^{(0)}, \boldsymbol{\rho}^{(2)}, \boldsymbol{\eta}$ and $\boldsymbol{\phi}$ are second-order tensors; $\boldsymbol{\mu}^{(1)}$ and $\boldsymbol{\psi}$ are third-order tensors, and $\boldsymbol{\mu}^{(2)}$ is a fourth-order tensor. 


\section{Effective boundary conditions for one-dimensional problems}

The subject of effective boundary conditions is, even for periodic media, highly challenging problem that admits explicit formulation only under special circumstances, see e.g. [13] and references therein. One such amenable class of problems is one-dimensional wave motion in bounded periodic domains where the effective boundary conditions, when expanded up to the second order [19, take the form of (elastic spring-like) Robin conditions [? ] written in terms of the mean field. This motivates our attempt to introduce the effective boundary conditions for quasi-periodic media as described next. To our knowledge, this problem has not been considered in the literature.

Let us consider a mixed boundary value problem (BVP) describing the shear wave motion in a quasiperiodic medium $Y=(0,1)$ that is fixed at $x=0$ and subjected to time-harmonic shear traction $\tau$ at $x=1$, see Fig. 2. With reference to (6), the BVP reads

$$
\begin{array}{ll}
\frac{\mathrm{d}}{\mathrm{d} x}\left(G(x) \frac{\mathrm{d} u}{\mathrm{~d} x}\right)+\rho(x) \omega^{2} u=0, & x \in Y, \\
u=0, & x=0, \\
\tau=G(x) \frac{\mathrm{d} u}{\mathrm{~d} x}, & x=1,
\end{array}
$$

where $G$ and $\rho$ are given by either $(2)$ or $(3)$.

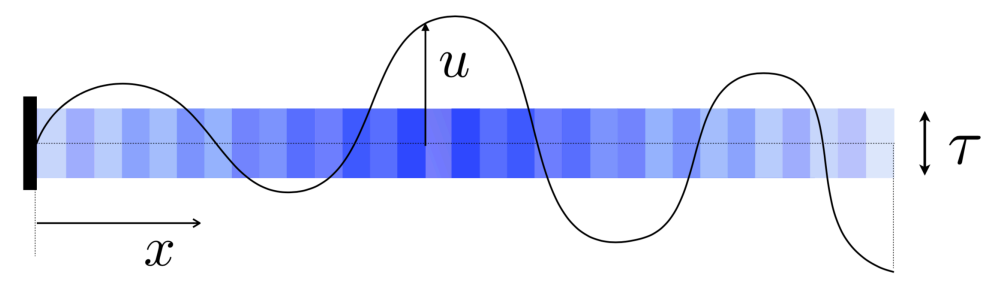

Figure 2: Shear waves in a finite quasi-periodic medium.

\subsection{Effective field equation}

To facilitate the development of effective boundary conditions, it is next useful to rewrite the second-order effective field equation (34) by expressing the featured third- and fourth-order derivatives in terms of their lower-order companions $\left(\langle u\rangle,\langle u\rangle_{, x},\langle u\rangle_{, x x}\right)$ using the $O(1)$ effective field equation 24 and the $O(\epsilon)$ effective field equation (29). Accordingly, (34) can be rewritten compactly as

$$
\left\{E_{5}(x)+\omega^{2} E_{3}(x)\right\}\langle u\rangle_{, x x}+\left\{E_{4}(x)+\omega^{2} E_{2}(x)\right\}\langle u\rangle_{, x}+\omega^{2} E_{1}(x)\langle u\rangle=O\left(\epsilon^{3}\right), \quad x \in \mathbb{R}
$$


where

$$
\begin{aligned}
E_{1}(x)= & \varrho^{(0)}+\epsilon\left(\rho^{(1)}-\mu^{(1)}\left[\frac{\rho^{(0)}}{\mu^{(0)}}\right]_{x}\right)+\epsilon^{2}\left(\mu ^ { ( 1 ) } \left\{\left[\frac{\rho^{(0)}}{\mu^{(0)}}\right]_{, x}\left(\frac{\mu_{, x}^{(1)}+\eta^{(1)}}{\mu^{(0)}}+\left[\frac{\mu^{(1)}}{\mu^{(0)}}\right]_{, x}\right)+\right.\right. \\
& \left.\left.\frac{\mu^{(1)}}{\mu^{(0)}}\left(\left[\frac{\rho^{(0)}}{\mu^{(0)}}\right]_{, x x}-\left[\frac{\mu_{, x}^{(0)}}{\mu^{(0)}}\right]\left[\frac{\rho^{(0)}}{\mu^{(0)}}\right]_{, x}\right)\right\}-\left[\frac{\varrho^{(0)}}{\mu^{(0)}}\right]_{x}\left(\psi+\mu_{, x}^{(2)}\right)+\mu^{(2)}\left\{\frac{\mu_{, x}^{(0)}}{\mu^{(0)}}\left[\frac{\varrho^{(0)}}{\mu^{(0)}}\right]_{x}-\left[\frac{\varrho^{(0)}}{\mu^{(0)}}\right]_{, x x}\right\}\right) \\
E_{2}(x)=- & \epsilon\left(\mu^{(1)}\left[\frac{\rho^{(0)}}{\mu^{(0)}}\right]\right)+\epsilon^{2}\left(\mu ^ { ( 1 ) } \left\{\left[\frac{\rho^{(0)}}{\mu^{(0)}}\right]\left(\left[\frac{\mu_{, x}^{(1)}+\eta^{(1)}}{\mu^{(0)}}\right]+\left[\frac{\mu^{(1)}}{\mu^{(0)}}\right]_{, x}\right)+\left[\frac{\mu^{(1)}}{\mu^{(0)}}\right]\left(2\left[\frac{\rho^{(0)}}{\mu^{(0)}}\right]_{, x}-\left[\frac{\mu_{, x}^{(0)}}{\mu^{(0)}}\right]\left[\frac{\rho^{(0)}}{\mu^{(0)}}\right]\right)\right.\right. \\
& \left.\left.-\left[\frac{\rho^{(1)}}{\mu^{(0)}}\right]_{, x}\right\}+\varrho^{(2)}-\frac{\varrho^{(0)}}{\mu^{(0)}}\left(\psi+\mu_{, x}^{(2)}\right)+\mu^{(2)}\left\{\left(\frac{\mu_{, x}^{(0)}}{\mu^{(0)}}\right)\left(\frac{\varrho^{(0)}}{\mu^{(0)}}\right)-2\left[\frac{\varrho^{(0)}}{\mu^{(0)}}\right]_{, x}\right\}\right) \\
E_{3}(x)= & \epsilon^{2}\left(\mu^{(1)}\left\{\left[\frac{\rho^{(0)}}{\mu^{(0)}}\right]\left[\frac{\mu^{(1)}}{\mu^{(0)}}\right]-\left[\frac{\rho^{(1)}}{\mu^{(0)}}\right]\right\}+\varrho^{(2)}-\mu^{(2)}\left(\frac{\varrho^{(0)}}{\mu^{(0)}}\right)\right) \\
E_{4}(x)= & \mu_{, x}^{(0)}+\epsilon\left(\eta_{, x}^{(1)}-\mu^{(1)}\left[\frac{\mu_{, x}^{(0)}}{\mu^{(0)}}\right]_{x}\right)+\epsilon^{2}\left(\phi_{, x}-\left[\frac{\mu_{, x}^{(0)}}{\mu^{(0)}}\right]_{, x}\left(\psi+\mu_{, x}^{(2)}\right)+\mu^{(2)}\left\{\left(\frac{\mu_{, x}^{(0)}}{\mu^{(0)}}\right)\left[\frac{\mu_{, x}^{(0)}}{\mu^{(0)}}\right]_{, x}-\left[\frac{\mu_{, x}^{(0)}}{\mu^{(0)}}\right]_{, x}\left(\left[\frac{\mu_{, x}^{(1)}+\eta^{(1)}}{\mu^{(0)}}\right]+\left[\frac{\mu^{(1)}}{\mu^{(0)}}\right]_{, x}\right)-\left[\frac{\eta_{, x}^{(1)}}{\mu^{(0)}}\right]_{, x}+\left[\frac{\mu^{(1)}}{\mu^{(0)}}\right]\left(\left[\frac{\mu_{, x}^{(0)}}{\mu^{(0)}}\right]_{, x x}-\left[\frac{\mu_{, x}^{(0)}}{\mu^{(0)}}\right]\left[\frac{\mu_{x}^{(0)}}{\mu^{(0)}}\right]_{, x}\right)\right\}\right) \\
E_{5}(x)= & \mu^{(0)}+\epsilon\left(\eta^{(1)}+\mu_{, x}^{(1)}-\mu^{(1)}\left[\frac{\mu_{, x}^{(0)}}{\mu^{(0)}}\right]\right)+\epsilon^{2}\left(\psi_{, x}+\phi-\frac{\mu_{, x}^{(0)}}{\mu^{(0)}}\left(\psi+\mu_{, x}^{(2)}\right)+\mu^{(2)}\left\{\left[\frac{\mu_{x}^{(0)}}{\mu^{(0)}}\right]^{2}-2\left[\frac{\mu_{x}^{(0)}}{\mu^{(0)}}\right]_{, x}\right\}\right. \\
& \left.+\mu^{(1)}\left\{\left[\frac{\mu_{, x}^{(0)}}{\mu^{(0)}}\right]\left(\left[\frac{\mu_{, x}^{(1)}+\eta^{(1)}}{\mu^{(0)}}\right]+\left[\frac{\mu^{(1)}}{\mu^{(0)}}\right]_{, x}\right)-\left[\frac{\eta_{, x}^{(1)}}{\mu^{(0)}}\right]-\left[\frac{\mu_{, x}^{(1)}+\eta^{(1)}}{\mu^{(0)}}\right]_{, x}+\left[\frac{\mu^{(1)}}{\mu^{(0)}}\right]\left(2\left[\frac{\mu_{, x}^{(0)}}{\mu^{(0)}}\right]_{, x}-\left[\frac{\mu_{, x}^{(0)}}{\mu^{(0)}}\right]^{2}\right)\right\}\right)
\end{aligned}
$$

\subsection{Zeroth-order model}

By virtue of (14), 17) and 42, , one finds that the zeroth-order, single-scale approximations of the displacement field $u(x)$ and stress field $\sigma(x)$ solving (54) can be written respectively as

$$
\begin{aligned}
u^{[0]}(x) & =\langle u\rangle^{[0]}, \\
\sigma^{[0]}(x) & =\mu^{(0)}(x)\langle u\rangle_{, x}^{[0]} \Sigma_{0}(x, x / \epsilon),
\end{aligned}
$$

where $\Sigma_{0}(x, x / \epsilon)=1$ as examined earlier. By requiring the mean-field equation 55 and the local boundary conditions in (54) to be satisfied up to $O(1)$, the mean field $\langle u\rangle^{[0]}$ can be shown to satisfy the effective BVP

$$
\begin{array}{ll}
\bar{E}_{5}(x)\langle u\rangle_{, x x}^{[0]}+\bar{E}_{4}(x)\langle u\rangle_{, x}^{[0]}+\omega^{2} \bar{E}_{1}(x)\langle u\rangle^{[0]}=0, & x \in Y \\
\langle u\rangle^{[0]}=0, & x=0, \\
\langle u\rangle_{, x}^{[0]}=\frac{\tau}{\mu^{(0)}(1)}, & x=1,
\end{array}
$$

where $\bar{E}_{1}, \bar{E}_{4}$, and $\bar{E}_{5}$ denote respectively the truncations of $E_{1}, E_{4}$, and $E_{5}$ that retain terms up to $O(1)$.

\subsection{First-order model}

By pursuing the analysis similar to that in Section 5.2 we next proceed with the first-order homogenized model. On recalling (14) and making use of (19) and (41), the first-order approximations of $u(x)$ and $\sigma(x)$ satisfying (54) can be written as

$$
\begin{aligned}
u^{[1]}(x) & =\langle u\rangle^{[1]}+\epsilon P(x, x / \epsilon)\langle u\rangle_{, x}^{[1]}, \\
\sigma^{[1]}(x) & =\mu^{(0)}(x)\left[\left(\Sigma_{0}(x, x / \epsilon)+\epsilon \Sigma_{2}(x, x / \epsilon)\right)\langle u\rangle_{, x}^{[1]}+\epsilon \Sigma_{1}(x, x / \epsilon)\langle u\rangle_{, x x}^{[1]}\right],
\end{aligned}
$$


Then, by requiring the mean-field equation (55) and the local boundary conditions in (54) to be satisfied up to $O(\epsilon)$, the mean field $\langle u\rangle^{[1]}$ can be shown to satisfy the effective BVP

$$
\begin{array}{lr}
\hat{E}_{5}(x)\langle u\rangle_{, x x}^{[1]}+\hat{E}_{4}(x)\langle u\rangle_{, x}^{[1]}+\omega^{2} \hat{E}_{1}(x)\langle u\rangle^{[1]}=0, & x \in Y, \\
\langle u\rangle^{[1]}+\epsilon P(0,0)\langle u\rangle_{, x}^{[1]}=0, & x=0, \\
\left\{-\epsilon \Sigma_{1}(1,1) \omega^{2} \frac{\bar{E}_{1}(1)}{\bar{E}_{5}(1)}\right\}\langle u\rangle^{[1]}+\left\{\Sigma_{0}(1,1)+\epsilon\left(\Sigma_{2}(1,1)-\Sigma_{1}(1,1) \frac{\bar{E}_{4}(1)}{\bar{E}_{5}(1)}\right)\right\}\langle u\rangle_{, x}^{[1]}=\frac{\tau}{\mu^{(0)}(1)}, & x=1,
\end{array}
$$

where $\hat{E}_{j}(j=\overline{1,5})$ denotes the truncation of $E_{j}$ that discards $O\left(\epsilon^{2}\right)$ correction terms. Note that in 60, we utilized the featured field equation $\langle u\rangle_{, x x}^{[1]}=-\hat{E}_{5}^{-1}\left(\omega^{2} \hat{E}_{1}\langle u\rangle^{[1]}+\hat{E}_{4}\langle u\rangle_{x}^{[1]}\right)$ in the stress boundary condition in order to obtain Robin-type boundary condition at each end.

\subsection{Second-order model}

Proceeding with the second-order homogenization, one can similarly write the second-order approximations of $u(x)$ and $\sigma(x)$ solving (54) as

$$
\begin{aligned}
u^{[2]}(x)= & \langle u\rangle^{[2]}+\left[\epsilon P(x, x / \epsilon)+\epsilon^{2} \tilde{P}(x, x / \epsilon)\right]\langle u\rangle_{, x}^{[2]}+\epsilon^{2} Q(x, x / \epsilon)\langle u\rangle_{, x x}^{[2]}, \\
\sigma^{[2]}(x)= & \mu^{(0)}(x)\left[\left(\Sigma_{0}(x, x / \epsilon)+\epsilon \Sigma_{2}(x, x / \epsilon)+\epsilon^{2} \Sigma_{3}(x, x / \epsilon)\right)\langle u\rangle_{, x}^{[2]}\right. \\
& \left.+\left(\epsilon \Sigma_{1}(x, x / \epsilon)+\epsilon^{2} \Sigma_{4}(x, x / \epsilon)\right)\langle u\rangle_{, x x}^{[2]}+\epsilon^{2} \Sigma_{5}(x, x / \epsilon)\langle u\rangle_{, x x x}^{[2]}\right],
\end{aligned}
$$

where the second-order mean field $\langle u\rangle^{[2]}$ solves the BVP

$$
\begin{array}{ll}
\left\{E_{5}(x)+\omega^{2} E_{3}(x)\right\}\langle u\rangle_{, x x}^{[2]}+\left\{E_{4}(x)+\omega^{2} E_{2}(x)\right\}\langle u\rangle_{, x}^{[2]}+\omega^{2} E_{1}(x)\langle u\rangle^{[2]}=0, & x \in Y, \\
\left\{1-\epsilon^{2} Q(0,0) \mathcal{D}(0) \mathcal{F}(0)\right\}\langle u\rangle^{[2]}+\left\{\epsilon P(0,0)+\epsilon^{2} \tilde{P}(0,0)-\epsilon^{2} Q(0,0) \mathcal{D}(0) \mathcal{E}(0)\right\}\langle u\rangle_{, x}^{[2]}=0, & x=0,{ }^{(02)} \\
\left\{\mathcal{C}(1,1) \mathcal{H}_{1}(1)-\mathcal{D}(1) \mathcal{B}(1,1) \mathcal{F}(1)\right\}\langle u\rangle^{[2]}+\left\{\mathcal{A}(1,1)+\mathcal{C}(1,1) \mathcal{H}_{2}(1)-\mathcal{D}(1) \mathcal{B}(1,1) \mathcal{E}(1)\right\}\langle u\rangle_{, x}^{[2]}=\frac{\tau}{\mu^{(0)}(1)}, & x=1,
\end{array}
$$

where

$$
\begin{aligned}
& \mathcal{A}(x, x / \epsilon)=\Sigma_{0}(x, x / \epsilon)+\epsilon \Sigma_{2}(x, x / \epsilon)+\epsilon^{2} \Sigma_{3}(x, x / \epsilon), \quad \mathcal{B}(x, x / \epsilon)=\epsilon \Sigma_{1}(x, x / \epsilon)+\epsilon^{2} \Sigma_{4}(x, x / \epsilon), \\
& \mathcal{C}(x, x / \epsilon)=\epsilon^{2} \Sigma_{5}(x, x / \epsilon), \quad \mathcal{D}(x)=\left(\omega^{2} E_{3}(x)+E_{5}(x)\right)^{-1}, \quad \mathcal{E}(x)=\omega^{2} E_{2}(x)+E_{4}(x), \quad \mathcal{F}(x)=\omega^{2} E_{1}(x), \\
& \mathcal{H}_{1}(x)=-\mathcal{D}_{, x} \mathcal{F}+\mathcal{D}^{2} \mathcal{E} \mathcal{F}-\mathcal{D} \mathcal{F}_{, x}, \\
& \mathcal{H}_{2}(x)=-\mathcal{D}_{, x} \mathcal{E}-\mathcal{D} \mathcal{E}_{, x}+\mathcal{D}^{2} \mathcal{E}^{2}-\mathcal{D} \mathcal{F}(x),
\end{aligned}
$$

Note that in 62 , we utilized the featured field equation $\langle u\rangle_{, x x}^{[2]}=-\mathcal{D}\left[\mathcal{F}\langle u\rangle^{[2]}+\mathcal{E}\langle u\rangle_{, x}^{[2]}\right]$ and its derivative $\langle u\rangle_{, x x x}^{[2]}=\mathcal{H}_{1}\langle u\rangle^{[2]}+\mathcal{H}_{2}\langle u\rangle_{, x}^{[2]}$ in the stress boundary condition in order to obtain Robin-type boundary condition at each end.

Remark 8. As can be seen from 62, a second-order mean-field approximation of the BVP (54) entails (i) second-order field equation with smooth coefficients, and (ii) Robin-type boundary conditions. The foregoing analysis can be easily generated to situations where the domain terminates within some $\epsilon Y$, which then affects the constant coefficients specifying the boundary conditions. 


\section{Numerical results}

To illustrate the utility of the foregoing homogenization framework, we consider one-dimensional wave motion (6) in a quasi-periodic medium endowed with bilaminate microstructure. For generality, we consider both (a) waves in an unbounded domain $\mathbb{R}$ - in terms of the heterogeneity-induced wave dispersion, and (b) waves in a bounded domain $Y$ - in terms of the waveforms generated by prescribed boundary excitation. With reference to (2), we consider several examples or quasi-periodic structures endowed with macroscopic variation

$$
\begin{array}{lll}
\text { linear variation: } & G^{\prime}(x)=1+\gamma_{\mathrm{G}} x, & \rho^{\prime}(x)=1+\gamma_{\rho} x \\
\text { sine variation: } & G^{\prime}(x)=1+\gamma_{\mathrm{G}} \sin \left(2 \pi x+\beta_{\mathrm{G}}\right), & \rho^{\prime}(x)=1+\gamma_{\rho} \sin \left(2 \pi x+\beta_{\rho}\right)
\end{array}
$$

and piecewise-constant microscopic fluctuation

$$
G^{\prime \prime}(y)=\delta_{\mathrm{G}}(-1+2 H(y-\alpha)), \quad \rho^{\prime \prime}(y)=\delta_{\rho}(-1+2 H(y-\alpha)), \quad 0<y<1
$$

where $H$ is the Heaviside function and $\alpha \in(0,1), \delta_{\mathrm{G}}$, and $\delta_{\rho}$ are prescribed constants.

Remark 9. As stated earlier, all quantities in this study are assumed to be normalized with respect to "suitable dimensional basis". In this section, the latter is given by the triplet $\left\{G_{\circ}, \rho_{\circ}, L_{\circ}\right\}$, where $G_{\circ}$ and $\rho_{\circ}$ correspond to the respective constant terms in the macroscopic variations (64) -65 of the shear modulus and mass density, while $L_{\circ}$ signifies the physical length of the unit cell $Y$.

\subsection{Effective coefficients}

By applying the analysis from Section 3 to the class $\sqrt{64}-66$ of quasi-periodic media and solving the germane boundary value problems (35)-40, the effective coefficients of homogenization can be computed from 25), (30), and 33. An analytical solution in terms of the cell functions $P, Q, \tilde{P}, \tilde{R}, \tilde{Q}$ and $R$ is sought via the symbolic manipulation platform Mathematica. Note that for any macroscopic variation of the quasi-periodic medium (2) with bilaminate microstructure (66), one can explicitly compute $\varrho^{(0)}, \mu^{(0)}, \varrho^{(1)}$ and $\mu^{(1)}$ as

$$
\begin{aligned}
& \varrho^{(0)}(x)=\rho^{\prime}(x)+\delta_{\mathrm{G}}\left(1-2 \alpha_{\mathrm{G}}\right), \quad \mu^{(0)}(x)=\frac{\left(G^{\prime}(x)-\delta_{\mathrm{G}}\right)\left(G^{\prime}(x)+\delta_{\mathrm{G}}\right)}{\alpha\left(G^{\prime}(x)+\delta_{\mathrm{G}}\right)+(1-\alpha)\left(G^{\prime}(x)-\delta_{\mathrm{G}}\right)}, \\
& \varrho^{(1)}(x)=0, \quad \mu^{(1)}(x)=0,
\end{aligned}
$$

see also [24, 46] in the context of periodic media. Expressions for the remaining effective coefficients such as $\eta(x)$ and $\phi(x)$ in the second-order model (34), however, are rather lengthy and will not be reported. Instead, a Mathematica code for their evaluation (assuming $640-660)$ is provided as electronic supplementary material. From (67) it is also interesting to observe that $\mu^{(1)}$ and $\varrho^{(1)}$ vanish identically, which is a well known property of all periodic structures [24, 46]. With reference to Table 1 summarizing the example material profiles considered, Fig. 3 plots the featured cell functions for Material 3, while Fig. 4 shows the distribution of the corresponding effective coefficients. 
Table 1: Quasi-periodic profiles used in numerical simulations.

\begin{tabular}{|l|c|c|c|c|c|c|c|}
\hline$G^{\prime}(x)$ and $\rho^{\prime}(x)$ & $\gamma_{G}$ & $\delta_{G}$ & $\beta_{G}$ & $\gamma_{\rho}$ & $\delta_{\rho}$ & $\beta_{\rho}$ & $\alpha$ \\
\hline \hline Material 1 $: 65$ & $1 / 5$ & $3 / 5$ & 0 & $1 / 5$ & $1 / 25$ & 0 & $1 / 2$ \\
\hline Material 2: 65 & $1 / 5$ & $1 / 5$ & 0 & 0 & 0 & 0 & $1 / 2$ \\
\hline Material $3: 65$ & $1 / 5$ & $1 / 5$ & 0 & $1 / 5$ & $1 / 5$ & 0 & $1 / 2$ \\
\hline Material $4: 65$ & $1 / 5$ & $2 / 5$ & $\pi / 2$ & $1 / 5$ & $1 / 5$ & $\pi / 2$ & $1 / 2$ \\
\hline Material $5: 640$ & $2 \pi / 5$ & $2 / 5$ & - & $2 \pi / 5$ & $1 / 5$ & - & $1 / 2$ \\
\hline
\end{tabular}

As reported in [46, 30, higher-order effective coefficients for both periodic and quasi-periodic structures are typically small in magnitude relative to their leading-order companions, and Fig. 4 confirms this observation. For the material profiles considered, it will be demonstrated that such behavior results in only a modest correction of the phase of the asymptotic solution, but potentially significant amplitude corrections.
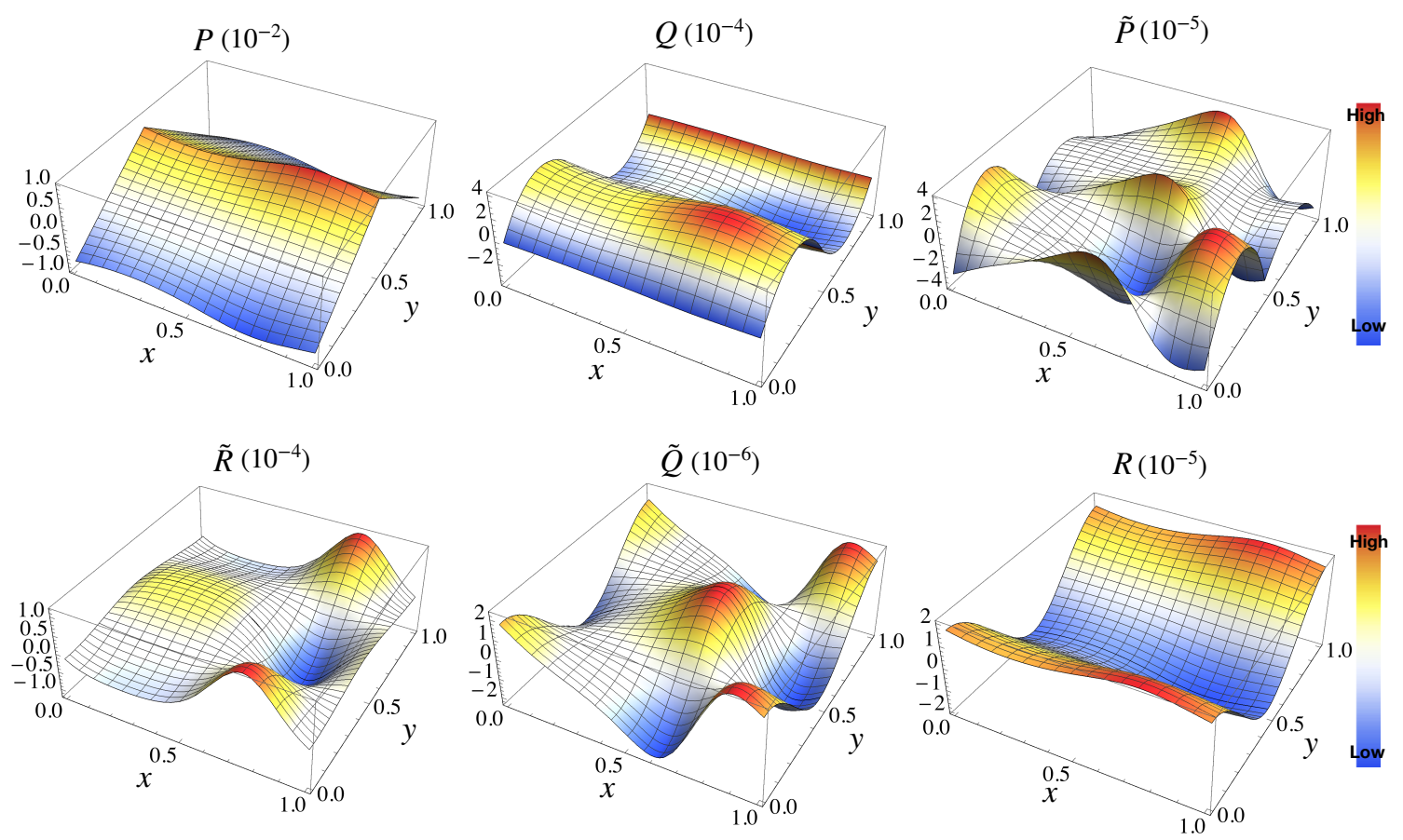

Figure 3: Cell functions $P, Q, \tilde{P}, \tilde{R}, \tilde{Q}$ and $R$ : Material 3, sinusoidal profile.

\subsection{Wave dispersion}

We next consider the native wave equation (11) for de facto periodic media where (i) $G^{\prime}$ and $\rho^{\prime}$ are $Y$-periodic, and (ii) $\epsilon=n^{-1}\left(n \in \mathbb{Z}^{+}\right)$. In this setting, we pursue the dispersion analysis via the Floquet-Bloch ap- 

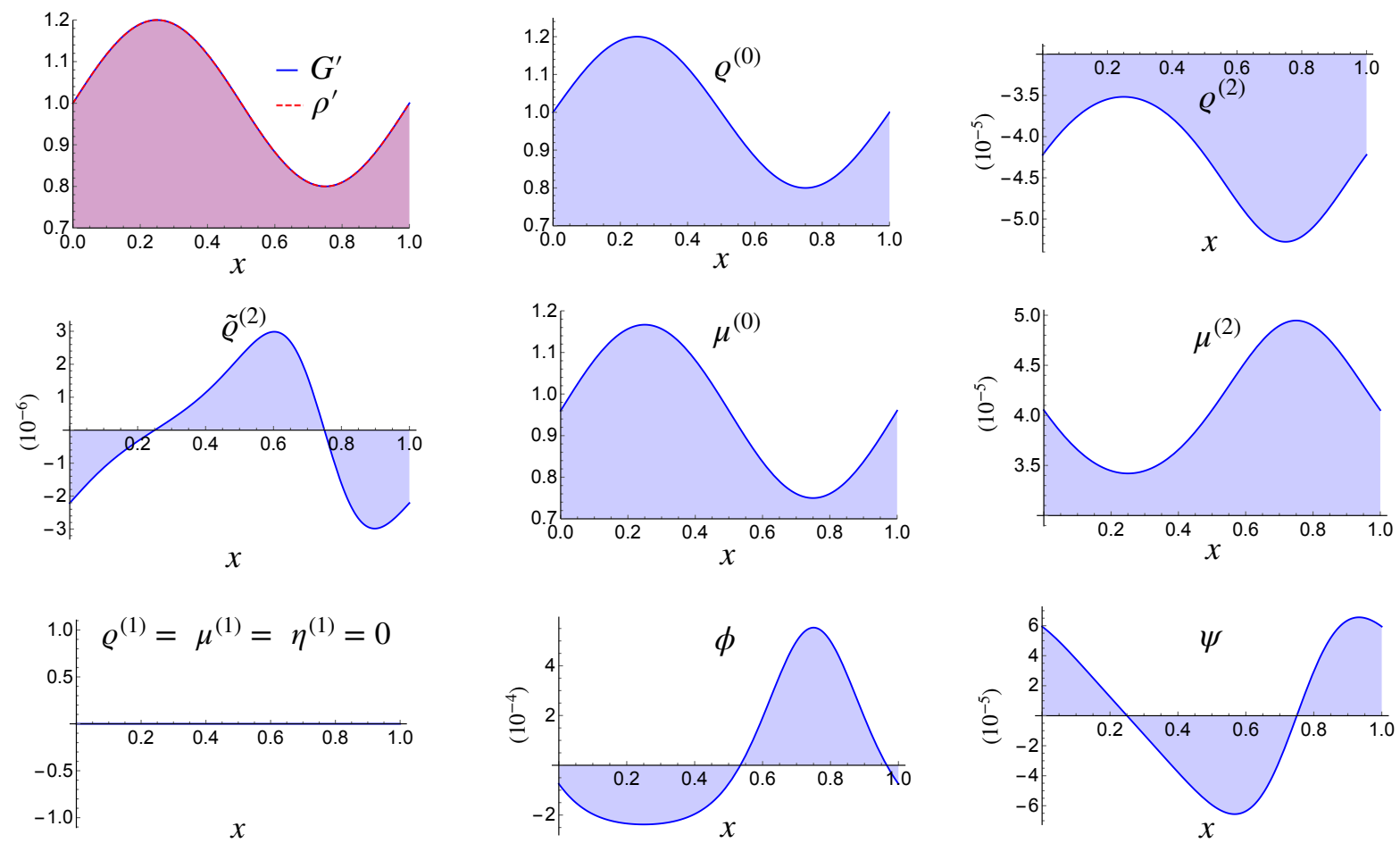

Figure 4: Macroscopic medium variations $\left(G^{\prime}, \rho^{\prime}\right)$ and effective coefficients $\varrho^{(0)}, \varrho^{(2)}, \varrho^{(2)}, \mu^{(0)}, \mu^{(2)}, \varrho^{(1)}, \mu^{(1)}, \eta, \phi$, and $\psi$ featured by the second-order model (53): Material 3, sinusoidal profile.

proach [29] by seeking a solution in the form

$$
u(x)=\tilde{u}(x) e^{i k x}, \quad \tilde{u}: Y \text {-periodic }
$$

where $Y=(0,1)$ as before, and $k$ is the wavenumber. Letting $\epsilon=n^{-1}$ for some $n>1$, it is clear that $G=G^{\prime}+G^{\prime \prime}$ and $\rho=\rho^{\prime}+\rho^{\prime \prime}$ are also $Y$-periodic. In this setting, we are in position to homogenize the "macrocell" $Y$ (containing $n$ periods of the microstructural variation) and compare the dispersion relationship computed in this way with numerical simulations of the native Floquet-Bloch problem given by (1) and (68). In what follows, we present the dispersion results for a sinusoidal macroscopic profile [65], illustrated schematically in Fig. 5 .

By way of (68), governing equation $(6)$ in $\mathbb{R}$ reduces to the macrocell problem

$$
\begin{aligned}
& \frac{\mathrm{d}}{\mathrm{d} x_{k}}\left(G(x) \frac{\mathrm{d} \tilde{u}}{\mathrm{~d} x_{k}}\right)+\rho(x) \omega^{2} \tilde{u}=0, \quad x \in Y \\
& \left.\tilde{u}\right|_{x=0}=\left.\tilde{u}\right|_{x=1},\left.\quad G \frac{\mathrm{d} \tilde{u}}{\mathrm{~d} x_{k}}\right|_{x=0}=-\left.G \frac{\mathrm{d} \tilde{u}}{\mathrm{~d} x_{k}}\right|_{x=1},
\end{aligned}
$$

where $\mathrm{d} / \mathrm{d} x_{k}:=\mathrm{d} / \mathrm{d} x+i k$. Hereon, we refer to a numerical solution of the boundary value problem (69) as the "exact" solution.

From 68), it follows that

$$
\langle u\rangle(x)=\langle\tilde{u}\rangle(x) e^{i k x}
$$




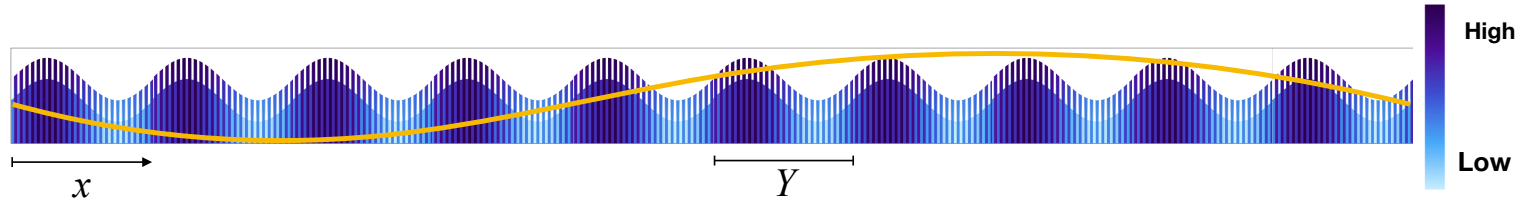

Figure 5: Long-wavelength shear waves propagating through an infinite periodic medium endowed with sinusoidal macroscopic profile 65 and bilaminate microstructure 66.

Assuming the material profile 65- 66 with $\epsilon=n^{-1}(n>1)$, one can make use of 70 together with the continuity and $Y$-periodicity of $E_{3}$ and $E_{5}$, to obtain the restriction of $(55)$ to $Y$ as

$$
\begin{gathered}
\omega^{2}\left\{E_{6}(x)\langle\tilde{u}\rangle+E_{7}(x)\langle\tilde{u}\rangle_{, x}+E_{3}(x)\langle\tilde{u}\rangle_{, x x}\right\}+E_{8}(x)\langle\tilde{u}\rangle+E_{9}(x)\langle\tilde{u}\rangle_{, x}+E_{5}(x)\langle\tilde{u}\rangle_{, x x}=0, \quad x \in Y \\
\left.\langle\tilde{u}\rangle\right|_{x=0}=\left.\langle\tilde{u}\rangle\right|_{x=1},\left.\quad\langle\tilde{u}\rangle_{, x}\right|_{x=0}=\left.\langle\tilde{u}\rangle_{, x}\right|_{x=1},
\end{gathered}
$$

where

$$
\begin{aligned}
& E_{6}(x)=E_{1}(x)+(i k) E_{2}(x)+(i k)^{2} E_{3}(x), \\
& E_{7}(x)=E_{2}(x)+2(i k) E_{3}(x), \\
& E_{8}(x)=(i k) E_{4}(x)+(i k)^{2} E_{5}(x), \\
& E_{9}(x)=E_{4}(x)+2(i k) E_{5}(x) .
\end{aligned}
$$

Since the macroscopic variation of the medium is now $Y$-periodic by design, we exploit the framework of Section 3 to obtain its (constant) leading-order effective coefficients, and we use the affiliated (linear) dispersion relationship as a baseline in the ensuing simulations.

To illustrate the analysis, we consider the wave dispersion in a "sinusoidal" medium 65 endowed with bilaminate microstructure (66) and $\epsilon=1 / 50$. To examine the performance of the effective model, the dispersion curves for the "exact" solution and its homogenized counterparts are obtained via the Floquet-Bloch approach applied to the "macrocell" $Y$. As a point of reference, Fig. 6 shows the "exact" dispersion curve in the first pass band (acoustic branch) for a medium composed of Material 1. To help parse the effects of macroscopic and microscopic heterogeneities on the overall behavior, we also include the dispersion curve for a microstructurefree medium with $G=G^{\prime}$ and $\rho=\rho^{\prime}$. From the display, one observes that the microscopic medium fluctuations have a remarkable effect on the overall wave dispersion.

Fig. 7 shows the dispersion curves in the first pass band for Material 3, and compares the exact model with its zeroth- and second-order asymptotic approximations stemming from (71). As a point of reference, included in the diagram is a linear dispersion relationship for the leading-order (non-dispersive) homogenized macrocell $Y$. The results suggest that the homogenized model is capable of capturing the exact solution with high accuracy. To highlight the dispersion effects, in Fig. 8 we recast the dispersion relationship in the terms of separation from the reference (non-dispersive) relationship for Material 2 and Material 3. From the displays, one observes that even the leading-order, homogenized model of the quasi-periodic medium stemming 
from (71) captures the exact dispersion with high accuracy, which puts in question the utility of the higherorder corrections. As will be seen shortly, however, the conclusion changes drastically when considering the solution of a BVP in terms of actual waveforms - that contain both amplitude and phase information.

For a complete insight into dispersive characteristics of the effective model, one can study the relative approximation error

$$
\text { Error }=\frac{\left|\omega^{(j)}-\omega^{(e)}\right|}{\left\|\omega^{(e)}\right\|_{2}}, \quad j=0,2
$$

where $\omega^{(e)}$ and $\omega^{(j)}$ denote respectively the exact and $j$ th-order homogenized solution, while $\|\cdot\|_{2}$ signifies the $L^{2}$-norm computed over the (positive half of the) first Brillouin zone. In this setting, Fig. 9 compares the relative error of the zeroth- and second-order effective models in describing the exact dispersion relationship for Material 2 and Material 3, which brings "under microscope" the improved fidelity brought about by the asymptotic correction.

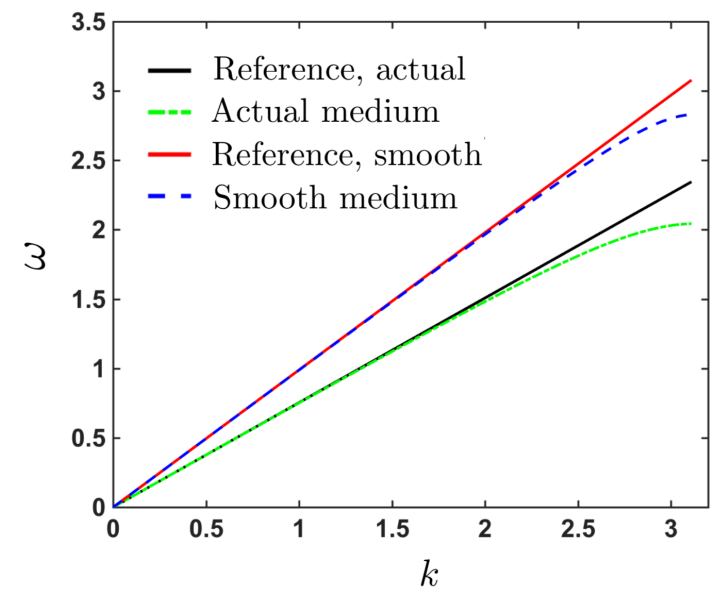

Figure 6: Exact dispersion relationship due to 69 for an infinite periodic medium composed of Material $1\left(G=G^{\prime}+G^{\prime \prime}\right.$ and $\rho=\rho^{\prime}+\rho^{\prime \prime}$, green solid line) versus that for its microstructure-free companion $\left(G=G^{\prime}\right.$ and $\rho=\rho^{\prime}$, blue dashed line). The fully homogenized, non-dispersive descriptions of $Y$ for both media (black solid line for Material 1 and red solid line for its microstructure-free counterpart) are included as baselines.

\subsection{Boundary value problem}

To complete the study, we consider an effective solution of the BVP (54) examined in Section 5 for the class of quasi-periodic media given by (64)-666). An "exact" solution of this problem is evaluated numerically via the propagator matrix approach using a high density of homogeneous sub-lamina to mimic the spatial variation of $G=G^{\prime}+G^{\prime \prime}$ and $\rho=\rho^{\prime}+\rho$ (see [46] for details in the context of periodic media). With reference to Table 1 . specifying the example material profiles, Table 2 completes the list of input parameters required to simulate the BVP. 


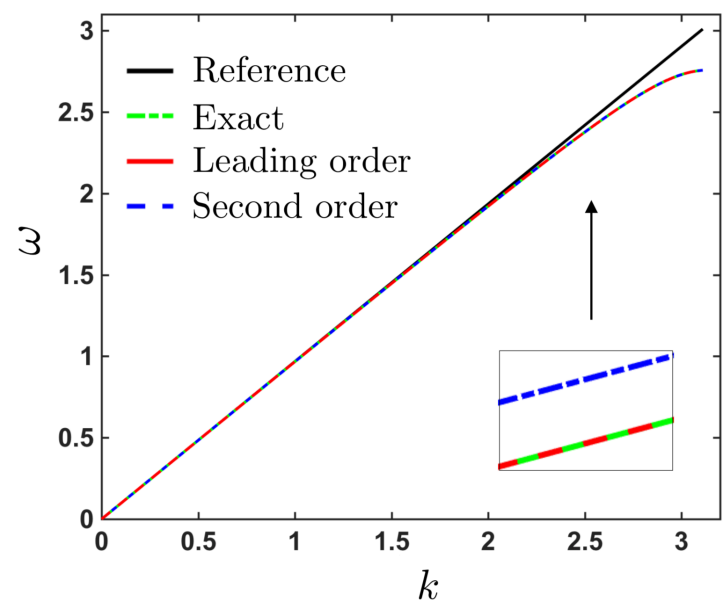

Figure 7: Exact dispersion relationship due to 69 for an infinite periodic medium composed of Material 3 (green solid line) versus its zeroth-order approximation (dot-dashed line) and second-order approximation (dashed line) according to 71).
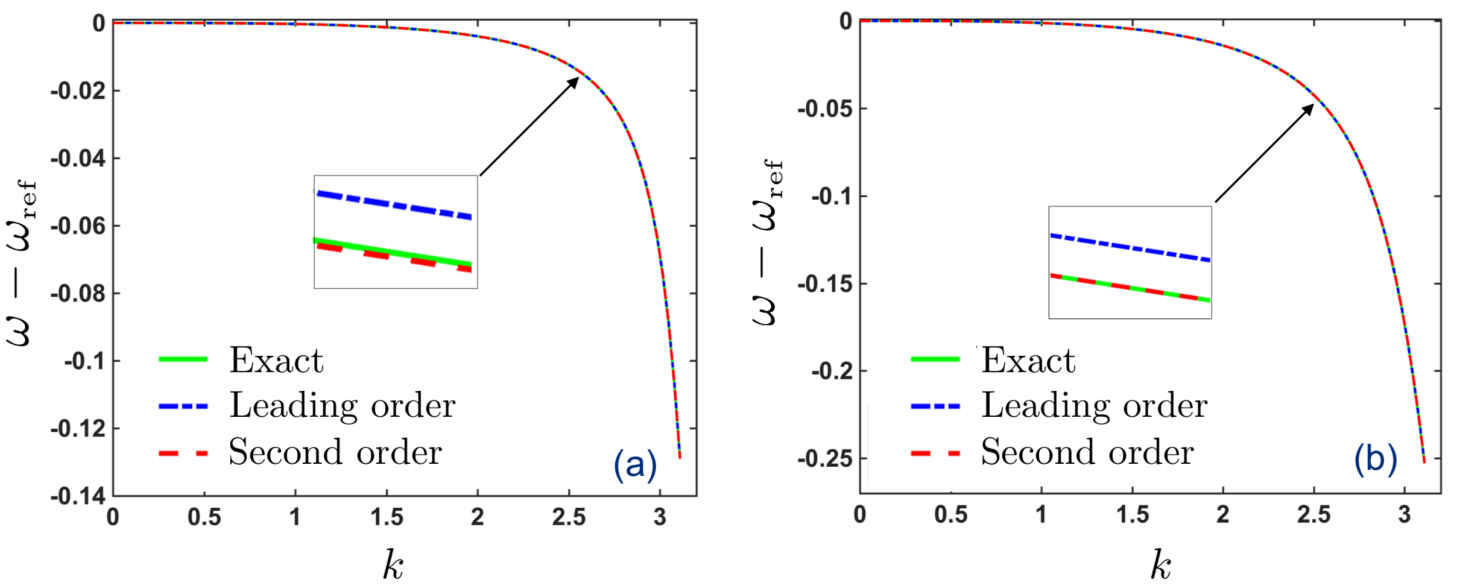

Figure 8: Separation from the reference non-dispersive model $\omega_{\text {ref }}(k)$ : exact solution (green dashed line), zeroth-order approximation (blue dashed-dot line), and second-order approximation (red dashed line) for an infinite periodic medium composed of (a) Material 2, and (b) Material 3.

Table 2: Parameters used for numerical simulation of the BVP 54 and its homogenized approximations 58, 60, and 62.

\begin{tabular}{|c|c|c|c|}
\hline Example & Material & $\omega$ & $\epsilon$ \\
\hline \hline Ex1 & 4 & $\pi^{2}$ & $1 / 20$ \\
\hline $\mathrm{Ex} 2$ & 4 & $3 \pi^{2}$ & $1 / 40$ \\
\hline $\mathrm{Ex} 3$ & 5 & $2 \pi^{2}$ & $1 / 20$ \\
\hline
\end{tabular}



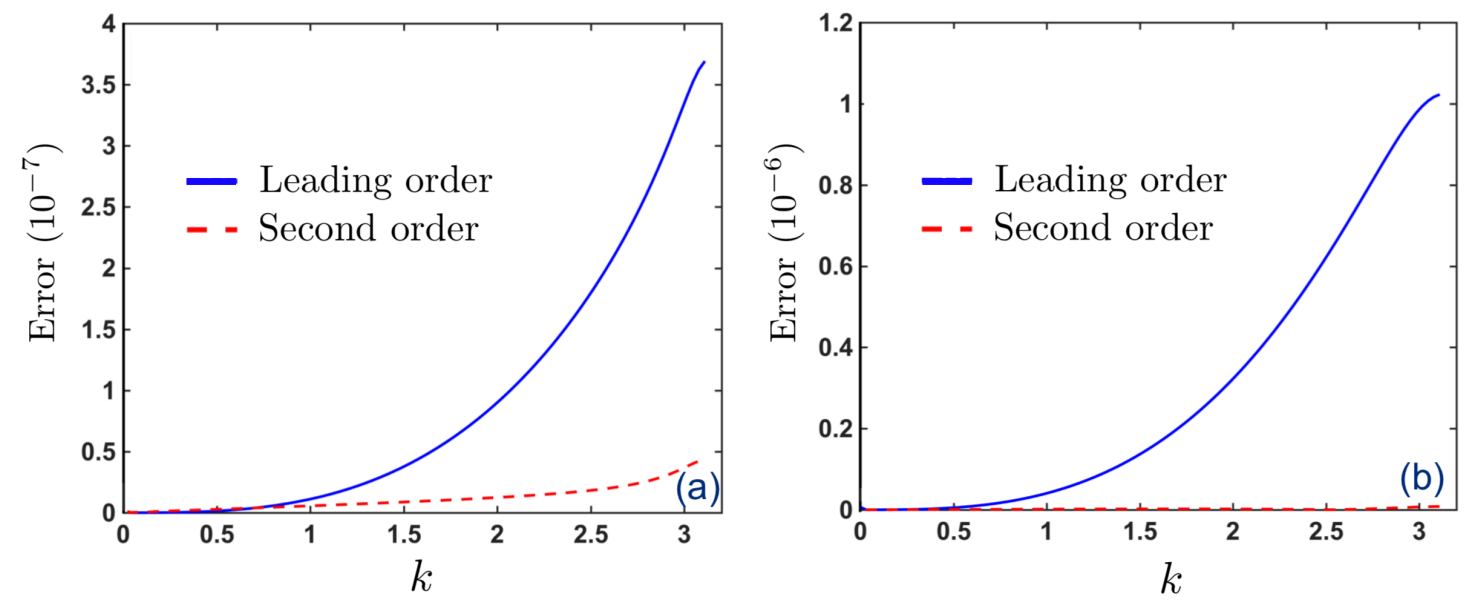

Figure 9: Relative error 733 of the asymptotic approximation $(j=0,2)$ for an infinite periodic medium composed of (a) Material 2, and (b) Material 3.

Remark 10. With reference to Fig. 7 describing the wave dispersion in an infinite periodic medium $\mathbb{R}$ composed of Material 3 (and thus that of Material 4), excitation frequencies listed in Table 2 may appear as being "too high" in that they are located beyond the first pass band. In this regard, it is important to recognize that the problems examined in Section 6.2 and Section 6.3 are fundamentally different. Specifically, Section 6.2 considers a macroscopically-periodic medium, that lends itself to the concept of the "macroscopic" Brillouin zone and allows for the computation of dispersion diagrams such as that in Fig. 7 . In contrast, this section is concerned with quasi-periodic media of finite extent, that are incompatible with the Bloch-wave representation (68) and the notion of the Brillouin zone. Instead, the key limitation on $\omega$ in our general study of quasi-periodic media (see Section 2.1) is that the lengthscale of microscopic fluctuations, $\epsilon Y$, is much smaller than the apparent wavelength. As will be seen shortly, all ensuing simulations meet this criterion by a safe margin. Concerning the remaining restriction on $\omega$ from Section 2.1. namely that the excitation frequency resides inside the first apparent "pass band", our numerical simulations show that increasing $\omega$ beyond the values listed in Table 2 may lead to the creation of an apparent band gap, manifested by an exponential decay of wave amplitude away from the loaded end, $x=1$. Similarly, a reduction in $\omega$ relative to the values listed in Table 2 can be shown (numerically) to result in a diminished error between that "exact" solution and its asymptotic approximation.

To illustrate the performance of the homogenized models, Fig. 10, Fig. 12 and Fig. 14 compare the mean fields $\langle u\rangle^{[0]},\langle u\rangle^{[1]}$, and $\langle u\rangle^{[2]}$ with the "exact" solution for examples Ex1, Ex2, and Ex3, respectively. As suggested earlier, the use of $\left(O(\epsilon)\right.$ and $\left.O\left(\epsilon^{2}\right)\right)$ asymptotic corrections is in this case critical to ensure the fidelity of the effective model. With such mean fields at hand, Fig. 11, Fig. 13 and Fig. 15 compare the 
full asymptotic approximations $u^{[0]}, u^{[1]}$, and $u^{[2]}$ (computed via (59) and (61)) with the "exact" solution in terms of both displacement and stress waveforms, respectively, for examples Ex1, Ex2, and Ex3. A common observation from these displays is that the second-order model provides a satisfactory description of the exact wavefield, whereas its lower-order companions appear to be deficient. This contrast is especially striking in terms of leading-order model $u^{[0]}=\langle u\rangle^{[0]}$ which appears to either undershoot by roughly $50 \%$, or overshoot by over $100 \%$, the "exact" solution. As mentioned earlier, however, all three approximations $\left(u^{[j]}, j=\overline{0,2}\right)$ are numerically observed to approach the "exact" solution as the excitation frequency $\omega$ is gradually decreased relative to the values listed in Table 2.

Remark 11. By comparing the respective zero crossings in Figs. 10 15, it is apparent that even the lowerorder models are quite good in capturing the phase of the solution - a result that is consistent with the findings of Section 6.2. However it is also clear that, at least for the frequencies selected, lower-order approximations are inadequate for synthesizing the actual waveforms in quasi-periodic media.

\section{Summary}

In this study, we pursue an effective description of the low-frequency wave motion in a macroscopically heterogeneous medium endowed with periodic microstructure. To this end, we deploy the framework of multiple scales and we apply the analysis to the scalar wave equation in one and multiple spatial dimensions. Through asymptotic expansion, the effective governing equation - free of microscopic fluctuations - is pursued up to the second order and shown to expose an intimate interplay between the dispersive effects of (periodic) micro-scale heterogeneities and their (generally non-periodic) macroscopic counterpart. More specifically, the germane low-frequency behavior is synthesized via a fourth-order differential equation (with smoothly varying coefficients) governing the mean wave motion in the medium, where the effect of microstructure is upscaled by way of the so-called cell functions. In an effort to demonstrate the relevance of our analysis toward solving boundary value problems, we also develop effective boundary conditions, up to the second order of asymptotic approximation, applicable to one-dimensional (1D) mean wave motion in a quasi-periodic medium. To our knowledge, this problem has escaped the scrutiny of earlier studies. We illustrate the analysis numerically in 1D by considering (i) low-frequency wave dispersion, (ii) mean-field homogenized description of waves propagating in a finite domain, and (iii) full-field homogenized description thereof. Specifically, we find that the microstructure may have a major effect on the overall wave dispersion in a quasi-periodic medium. In contrast to (i), however, where the latter appears to be well captured even by the leading-order model, the results in (ii) and (iii) illustrate the critical role that higher-order corrections may have in maintaining the fidelity of homogenized waveform description. 

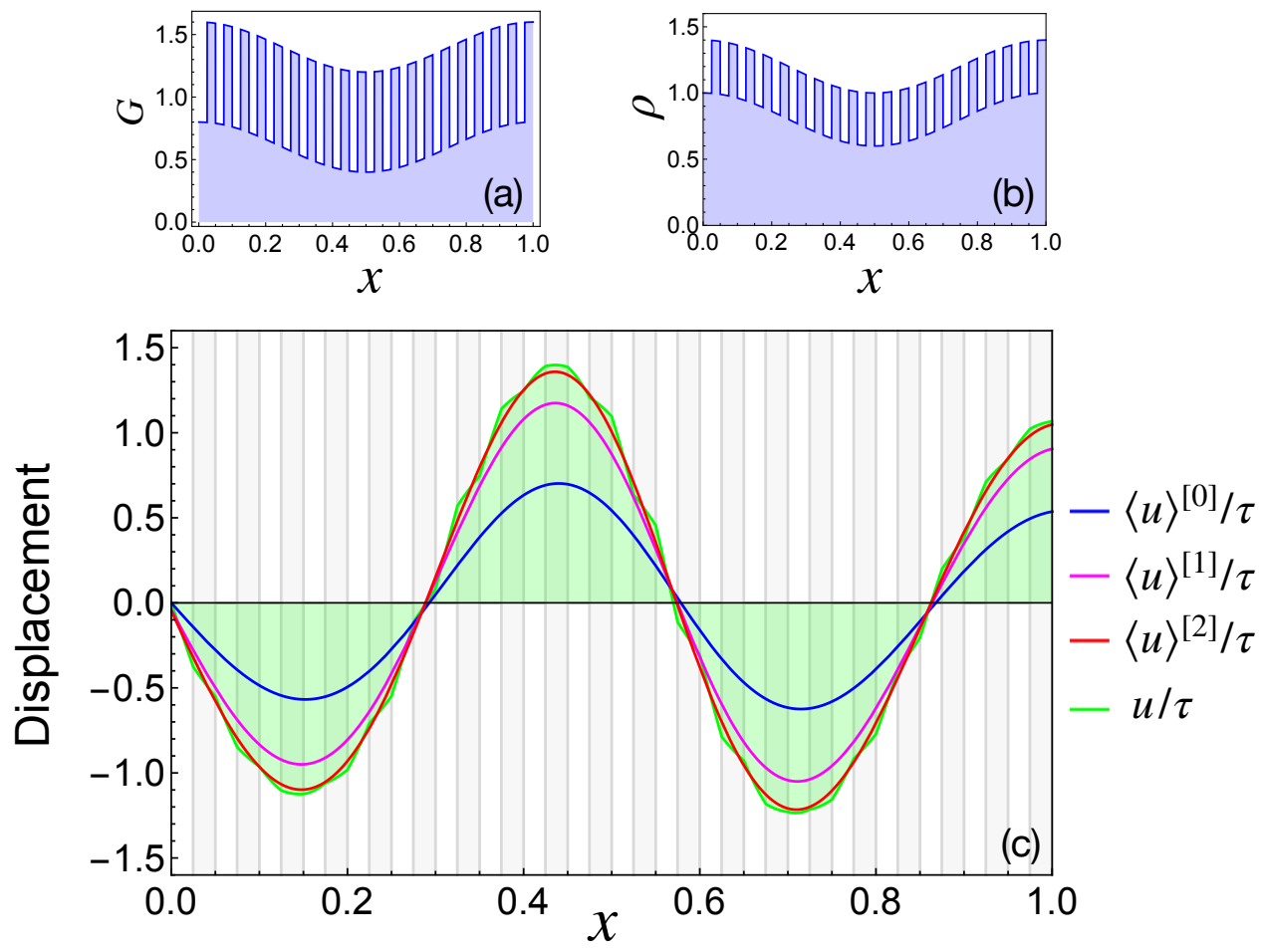

Figure 10: Example Ex1: (a) Shear modulus profile, (b) mass density profile, and (c) "exact" wave motion $u$ versus homogenized mean fields $\langle u\rangle^{[0]},\langle u\rangle^{[1]}$, and $\langle u\rangle^{[2]}$ for the BVP $[54$.

\section{Acknowledgements}

This work was supported in part through the endowed Shimizu Professorship, and Sommerfeld Fellowship to DS (Department of Civil, Environmental, and Geo- Engineering, University of Minnesota). The support provided by the Minnesota Supercomputing institute is kindly acknowledged. DS would also like to thank Othman Oudghiri-Idrissi for fruitful discussions and remarks.

\section{Appendix A. Cell functions describing effective wave motion in $\mathbb{R}^{d}(d>1)$}

With reference to (4), consider the situation where the unit cell $Y$ is composed of $N$ subdomains $Y_{q}(q=\overline{1, N})$ such that $G^{\prime \prime}(\boldsymbol{y})$ and $\rho^{\prime \prime}(\boldsymbol{y})$ according to either (2) or (3) vary smoothly within each $Y_{q}$. In this setting, one finds that the (zero-mean) cell functions $\boldsymbol{P} \in \mathbb{R}^{d}, \boldsymbol{Q} \in \mathbb{R}^{d \times d}, \boldsymbol{R} \in \mathbb{R}^{d \times d \times d}, \tilde{\boldsymbol{P}} \in \mathbb{R}^{d}, \tilde{\boldsymbol{Q}} \in \mathbb{R}^{d \times d}$ and $\tilde{\boldsymbol{R}} \in \mathbb{R}^{d}$ specifying the effective tensor coefficients in (53) according to [48), 500 and (52) solve the respective boundary value problems

$$
\begin{array}{ll}
\nabla_{\boldsymbol{y}} \cdot\left[G\left(\boldsymbol{I}_{2}+\nabla_{\boldsymbol{y}} \boldsymbol{P}\right)\right]=\mathbf{0}, \quad \boldsymbol{y} \in Y_{q} & \\
\boldsymbol{P}, G \boldsymbol{n} \cdot\left(\boldsymbol{I}_{2}+\nabla_{\boldsymbol{y}} \boldsymbol{P}\right), \quad Y \text {-periodic; } & \langle\boldsymbol{P}\rangle=\mathbf{0} \\
\llbracket \boldsymbol{P} \rrbracket=\mathbf{0}, \quad \llbracket G \boldsymbol{n} \cdot\left(\boldsymbol{I}_{2}+\nabla_{\boldsymbol{y}} \boldsymbol{P}\right) \rrbracket=\mathbf{0}, \quad \boldsymbol{y} \in \partial Y_{q} \backslash \partial Y ;
\end{array}
$$



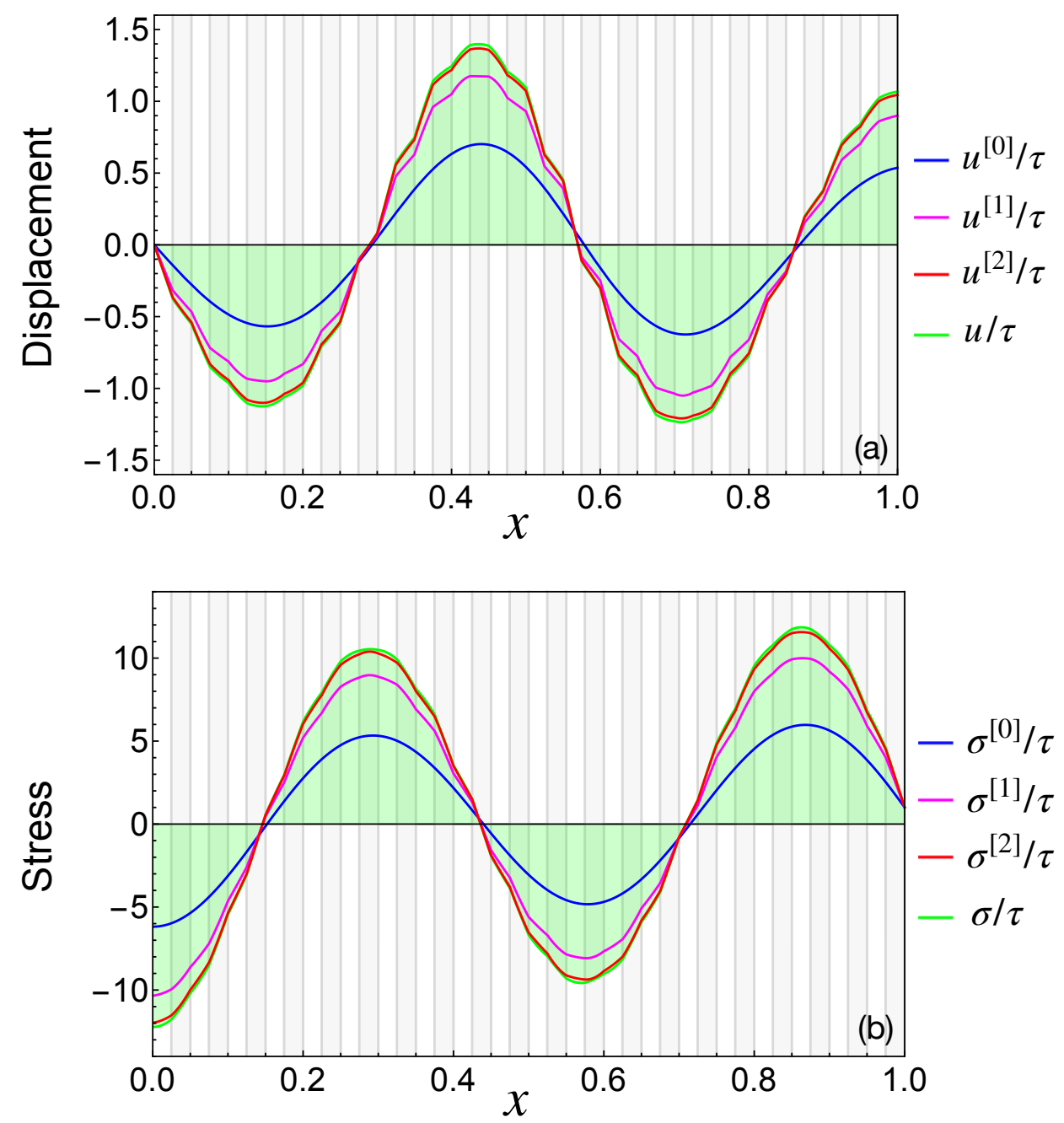

Figure 11: Example Ex1: (a) "exact" wave motion $u$ versus homogenized approximations $u^{[0]}, u^{[1]}$, and $u^{[2]}$; (b) Exact stress filed $\sigma(x)$ versus homogenized approximations $\sigma^{[0]}, \sigma^{[1]}$, and $\sigma^{[2]}$, for the BVP 54 .

$$
\begin{gathered}
\nabla_{\boldsymbol{y}}\left[G\left(\boldsymbol{P}+\nabla_{\boldsymbol{y}} \cdot \boldsymbol{Q}\right)\right]=\frac{\rho}{\varrho^{(0)}} \boldsymbol{\mu}^{(0)}-G\left(\boldsymbol{I}_{2}+\nabla_{\boldsymbol{y}} \boldsymbol{P}\right), \quad \boldsymbol{y} \in Y_{q} \\
\boldsymbol{Q}, G \boldsymbol{n} \cdot\left(\boldsymbol{P}+\nabla_{\boldsymbol{y}} \cdot \boldsymbol{Q}\right), \quad Y \text {-periodic; } \quad\langle\boldsymbol{Q}\rangle=\mathbf{0} \\
\llbracket \boldsymbol{Q} \rrbracket=\mathbf{0}, \quad \llbracket G \boldsymbol{n} \cdot\left(\boldsymbol{P}+\nabla_{\boldsymbol{y}} \cdot \boldsymbol{Q}\right) \rrbracket=\mathbf{0}, \quad \boldsymbol{y} \in \partial Y_{q} \backslash \partial Y ; \\
\nabla_{\boldsymbol{y}} \cdot\left[G\left(\nabla_{\boldsymbol{x}} \boldsymbol{P}+\nabla_{\boldsymbol{y}} \tilde{\boldsymbol{P}}\right)\right]=\frac{\rho}{\varrho^{(0)}} \nabla \cdot \boldsymbol{\mu}^{(0)}-\nabla_{\boldsymbol{x}} \cdot\left[G\left(\boldsymbol{I}_{2}+\nabla_{\boldsymbol{y}} \boldsymbol{P}\right)\right], \quad \boldsymbol{y} \in Y_{q} \\
\tilde{\boldsymbol{P}}, G \boldsymbol{n} \cdot\left(\nabla_{\boldsymbol{x}} \boldsymbol{P}+\nabla_{\boldsymbol{y}} \tilde{\boldsymbol{P}}\right), \quad Y \text {-periodic; } \quad\langle\tilde{\boldsymbol{P}}\rangle=\mathbf{0} \\
\llbracket \tilde{\boldsymbol{P}} \rrbracket=\mathbf{0}, \quad \llbracket G \boldsymbol{n} \cdot\left(\nabla_{\boldsymbol{x}} \boldsymbol{P}+\nabla_{\boldsymbol{y}} \tilde{\boldsymbol{P}}\right) \rrbracket=\mathbf{0}, \quad \boldsymbol{y} \in \partial Y_{q} \backslash \partial Y ;
\end{gathered}
$$



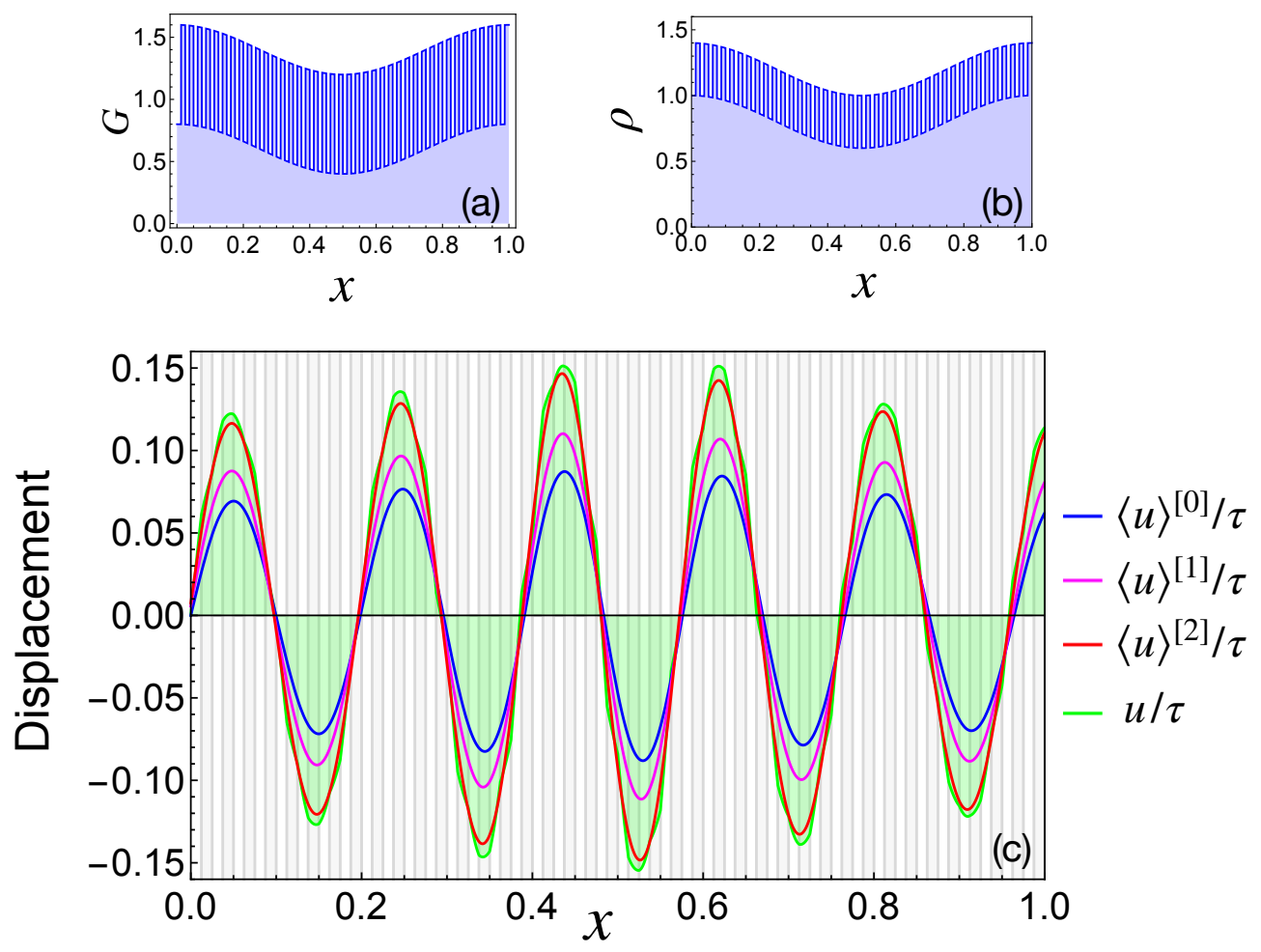

Figure 12: Example Ex2: (a) Shear modulus profile, (b) mass density profile, and (c) "exact" wave motion $u$ versus homogenized mean fields $\langle u\rangle^{[0]},\langle u\rangle^{[1]}$, and $\langle u\rangle^{[2]}$ for the BVP [54].

$$
\begin{aligned}
\nabla_{\boldsymbol{y}}\left[G\left(\tilde{\boldsymbol{P}}+\nabla_{\boldsymbol{x}} \cdot \boldsymbol{Q}+\nabla_{\boldsymbol{y}} \cdot \tilde{\boldsymbol{Q}}\right)\right]=\frac{\rho}{\varrho^{(0)}}\left(\boldsymbol{\eta}+\nabla \cdot \boldsymbol{\mu}^{(1)}\right) & -\nabla_{\boldsymbol{x}}\left[G\left(\boldsymbol{P}+\nabla_{\boldsymbol{y}} \cdot \boldsymbol{Q}\right)\right]-G\left(\nabla_{\boldsymbol{x}} \boldsymbol{P}+\nabla_{\boldsymbol{y}} \tilde{\boldsymbol{P}}\right) \\
& +\rho\left(\boldsymbol{P}-\frac{\varrho^{(1)}}{\varrho^{(0)}}\right) \otimes\left(\frac{\nabla \cdot \boldsymbol{\mu}^{(0)}}{\varrho^{(0)}}+\nabla \cdot\left[\frac{\boldsymbol{\mu}^{\mathbf{0}}}{\varrho^{(0)}}\right]\right), \quad \boldsymbol{y} \in Y_{q}
\end{aligned}
$$

$\tilde{\boldsymbol{Q}}, G \boldsymbol{n} \cdot\left(\tilde{\boldsymbol{P}}+\nabla_{\boldsymbol{x}} \cdot \boldsymbol{Q}+\nabla_{\boldsymbol{y}} \cdot \tilde{\boldsymbol{Q}}\right), \quad Y$-periodic; $\quad\langle\tilde{\boldsymbol{Q}}\rangle=\mathbf{0}$

$\llbracket \tilde{\boldsymbol{Q}} \rrbracket=\mathbf{0}, \quad \llbracket G \boldsymbol{n} \cdot\left(\tilde{\boldsymbol{P}}+\nabla_{\boldsymbol{x}} \cdot \boldsymbol{Q}+\nabla_{\boldsymbol{y}} \cdot \tilde{\boldsymbol{Q}}\right) \rrbracket=\mathbf{0}, \quad \boldsymbol{y} \in \partial Y_{q} \backslash \partial Y$

$$
\begin{aligned}
& \nabla_{\boldsymbol{y}}\left[G\left(\boldsymbol{Q}+\nabla_{\boldsymbol{y}} \cdot \boldsymbol{R}\right)\right]=\frac{\rho}{\varrho^{(0)}} \boldsymbol{\mu}^{(1)}-G\left(\boldsymbol{I}_{2} \otimes \boldsymbol{P}+\nabla_{\boldsymbol{y}} \boldsymbol{Q}\right)+\rho \frac{\boldsymbol{\mu}^{(0)}}{\varrho^{(0)}} \otimes\left(\boldsymbol{P}-\frac{\varrho^{(1)}}{\varrho^{(0)}}\right), \quad \boldsymbol{y} \in Y_{q} \\
& \boldsymbol{R}, G \boldsymbol{n} \cdot\left(\boldsymbol{Q}+\nabla_{\boldsymbol{y}} \cdot \boldsymbol{R}\right), \quad Y \text {-periodic; } \quad\langle\boldsymbol{R}\rangle=\mathbf{0} \\
& \llbracket \boldsymbol{R} \rrbracket=\mathbf{0}, \quad \llbracket G \boldsymbol{n} \cdot\left(\boldsymbol{Q}+\nabla_{\boldsymbol{y}} \cdot \boldsymbol{R}\right) \rrbracket=\mathbf{0}, \quad \boldsymbol{y} \in \partial Y_{q} \backslash \partial Y,
\end{aligned}
$$

and

$$
\begin{aligned}
& \nabla_{\boldsymbol{y}} \cdot\left[G\left(\nabla_{\boldsymbol{x}} \tilde{\boldsymbol{P}}+\nabla_{\boldsymbol{y}} \tilde{\boldsymbol{R}}\right)\right]=\frac{\rho}{\varrho^{(0)}} \nabla \cdot \boldsymbol{\eta}-\nabla_{\boldsymbol{x}} \cdot\left[G\left(\nabla_{\boldsymbol{x}} \boldsymbol{P}+\nabla_{\boldsymbol{y}} \tilde{\boldsymbol{P}}\right)\right]+\rho\left(\boldsymbol{P}-\frac{\varrho^{(1)}}{\varrho^{(0)}}\right) \nabla \cdot\left[\frac{\nabla \cdot \boldsymbol{\mu}^{\mathbf{0}}}{\varrho^{(0)}}\right], \quad \boldsymbol{y} \in Y_{q} \\
& \tilde{\boldsymbol{R}}, G \boldsymbol{n} \cdot\left(\nabla_{\boldsymbol{x}} \tilde{\boldsymbol{P}}+\nabla_{\boldsymbol{y}} \tilde{\boldsymbol{R}}\right), \quad Y \text {-periodic; } \quad\langle\tilde{\boldsymbol{R}}\rangle=\mathbf{0} \\
& \llbracket \tilde{\boldsymbol{R}} \rrbracket=\mathbf{0}, \quad \llbracket G \boldsymbol{n} \cdot\left(\nabla_{\boldsymbol{x}} \tilde{\boldsymbol{P}}+\nabla_{\boldsymbol{y}} \tilde{\boldsymbol{R}}\right) \rrbracket=\mathbf{0}, \quad \boldsymbol{y} \in \partial Y_{q} \backslash \partial Y .
\end{aligned}
$$



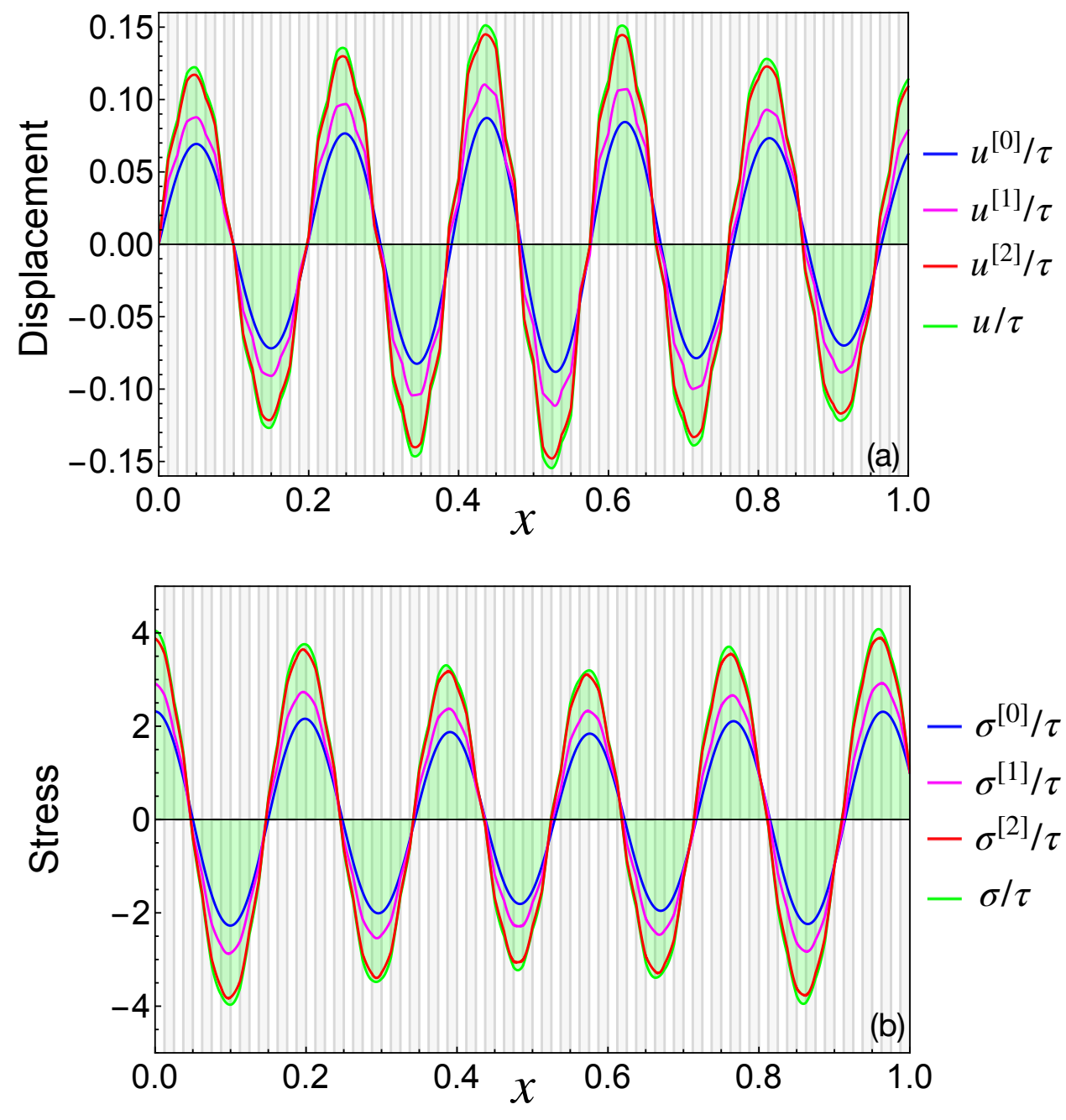

Figure 13: Example Ex2: (a) "exact" wave motion $u$ versus homogenized approximations $u^{[0]}, u^{[1]}$, and $u^{[2]}$; (b) "exact" stress filed $\sigma(x)$ versus homogenized approximations $\sigma^{[0]}, \sigma^{[1]}$, and $\sigma^{[2]}$, for the BVP 54 .

\section{References}

[1] H.L. Bertoni, L. S. Cheo, and T. Tamir. Frequency-selective reflection and transmission by a periodic dielectric layer. IEEE Trans. Antennas Propag., 37(78-83), 1989.

[2] R.A. Shelby. Experimental verification of a negative index of refraction. Science, pages 29277-79, 2001.

[3] I.V. Andrianov, J. Awrejcewicz, and A. A. Diskovsky. Homogenization of quasi-periodic structures. Journal of Vibration and Acoustics, 128(4):532-534, 2006.

[4] I.V. Andrianov, V.I. Bolshakov, V.V. Danishevskyy, and D. Weichert. Higher order asymptotic homogenization and wave propagation in periodic composite materials. Proc. R. Soc. A, 464:1181-1201, 2008. 

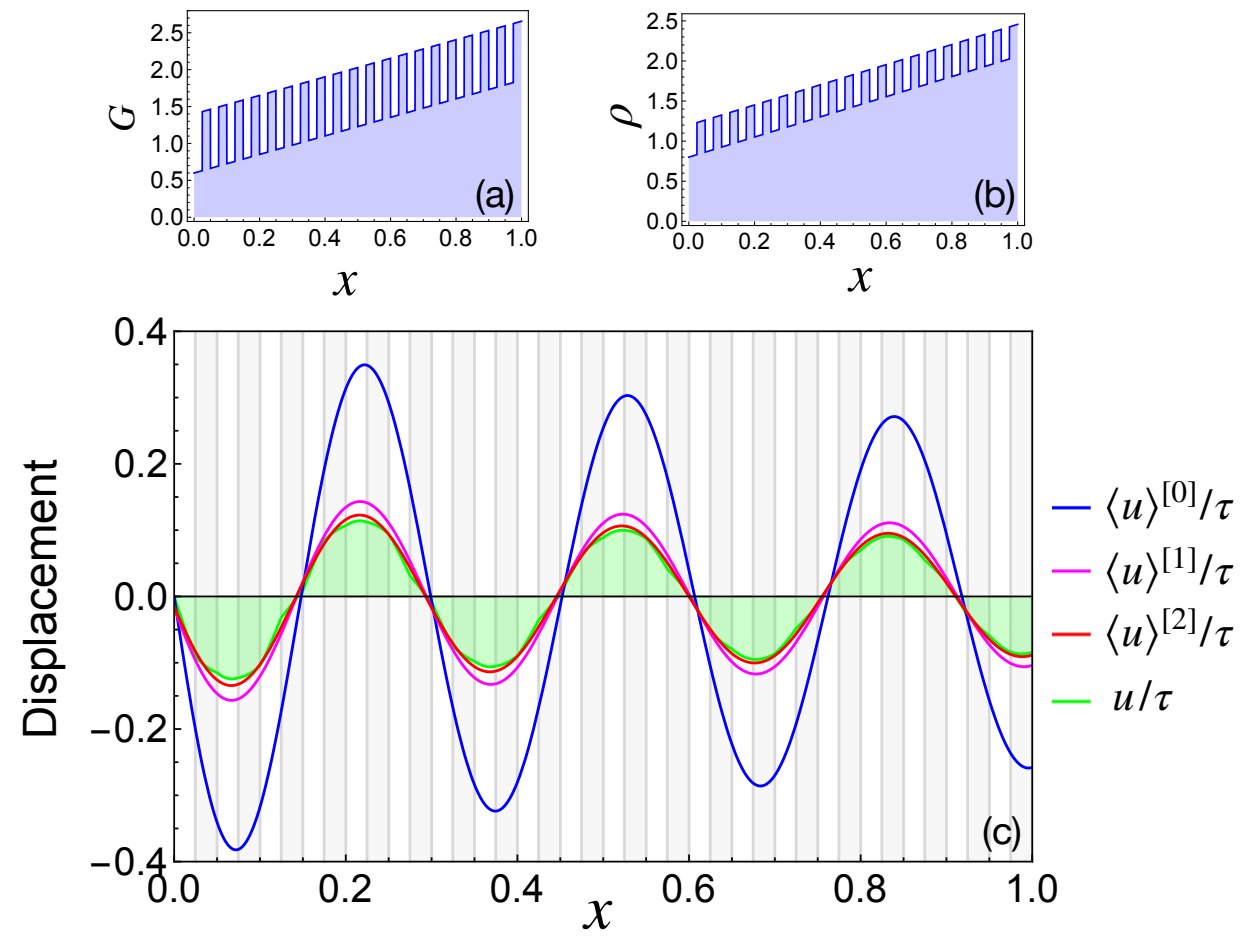

Figure 14: Example Ex3: (a) Shear modulus profile, (b) mass density profile, and (c) "exact" wave motion $u$ versus homogenized mean fields $\langle u\rangle^{[0]},\langle u\rangle^{[1]}$, and $\langle u\rangle^{[2]}$ for the BVP [54

[5] I. Babuska. Homogenization approach in engineering. Lectures Notes in Economics and Mathematical Systems, 134:137-153, 1976.

[6] N. Bakhvalov and G. Panasenko. Homogenisation: averaging processes in periodic media: mathematical problems in the mechanics of composite materials. Kluwer Academic Publishers, 1989.

[7] N.S. Bakhvalov and M.E. Eglit. Equations of higher order of accuracy describing the vibrations of thin plates. Prikl. Mat. Mekh., 69:656-675, 2005.

[8] N.S. Bakhvalov and M.E. Eglit. High-order accurate equations describing vibrations of thin bars. Comput. Mech. Math. Phys., 46:437-452, 2006.

[9] A. Bensoussan, J.L. Lions, and G. Papanicolaou. Asymptotic Analysis for Periodic Structures. NorthHolland, 1978.

[10] F. Bloch. Über die quantenmechanik der elektronen in kristallgittern. Z. Phys., 52:555-600, 1929.

[11] B. Boutin and J.L. Auriault. Rayleigh scattering in elastic composite materials. Int. J. Eng. Sci., 31:1669-1689, 1993. 

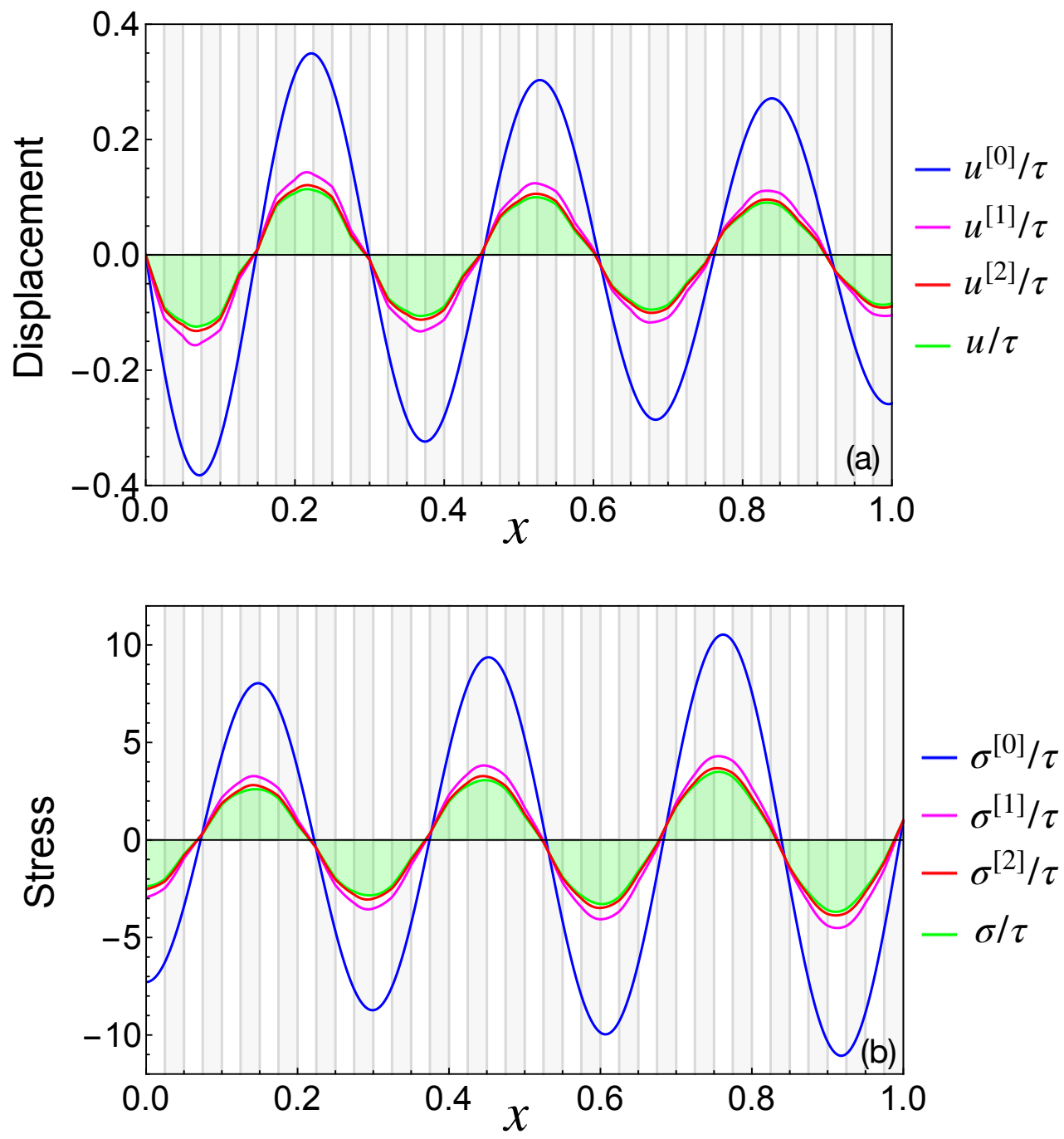

Figure 15: Example Ex3: (a) "exact" wave motion $u(x)$ versus homogenized approximations $u^{[0]}, u^{[1]}$, and $u^{[2]}$; (b) "exact" stress filed $\sigma(x)$ versus homogenized approximations $\sigma^{[0]}, \sigma^{[1]}$, and $\sigma^{[2]}$, for the BVP [54.

[12] L. Brillouin. Wave Propagation in Periodic Structures, 2nd edition. Dover Phoenix Editions New York, 2003.

[13] F. Cakoni, B.B. Guzina, S. Moskow, and T. Pangburn. Scattering by a bounded highly oscillating periodic medium and the effect of boundary correctors. SIAM Journal on Applied Mathematics, 79:1448-1474, 2019.

[14] L. Cao and J. Cui. Homogenization method for the quasi-periodic structures of composite materials. Math. Numer. Sin., 21:331-344, 1999.

[15] Y. Capdeville, L. Guillot, and J. J. Marigo. 2-D non-periodic homogenization to upscale elastic media for P-SV waves. Geophysical Journal International, 2(182):903-922, 2010. 
[16] P. Celli, B. Yousefzadeh, C. Daraio, and S. Gonella. Bandgap widening by disorder in rainbow metamaterials. Applied Physics Letters, 114:091903, 2019.

[17] W. Chen and J. Fish. A dispersive model for wave propagation in periodic heterogeneous media based on homogenization with multiple spatial and temporal scales. J. Appl. Mech. ASME, 68:153-161, 2001.

[18] A. Colombi, D. Colquitt, P. Roux, S. Guenneau, and R.V. Craster. A seismic metamaterial: The resonant metawedge. Scientific Reports, 6(1):1-6, 2016.

[19] R. Cornaggia and B.B. Guzina. Second-order homogenization of boundary and transmission conditions for one-dimensional waves in periodic media. Int. J. Solids Struct., 188-89:88-102, 2020.

[20] Richard V Craster, Julius Kaplunov, and Aleksey V Pichugin. High-frequency homogenization for periodic media. Proceedings of the Royal Society A: Mathematical, Physical and Engineering Sciences, 466(2120):2341-2362, 2010.

[21] P. Cupillard and Y. Capdeville. Non-periodic homogenization of 3D elastic media for the seismic wave equation. Geophysical Journal International, 213:983-1001, 2018.

[22] H. Dong, Y.F. Nie, J.Z. Cui, Y.T. Wu, and Z.H. Yang. Second-order two-scale analysis and numerical algorithm for the damped wave equations of composite materials with quasi- periodic structures. Appl. Math. Comput., 298:201-220, 2017.

[23] S. Fang, C. J. Zhi, X. Zhan, and D. Q. Li. A second-order and two-scale computation method for the quasi-periodic structures of composite materials. Finite Elements in Analysis and Design, 46:320-327, 2010.

[24] J. Fish and W. Chen. Higher-order homogenization of initial/boundary-value problem. J. Eng. Mech. ASCE, 127:1223-1230, 2001.

[25] J. Fish and W. Chen. Space-time multiscale model for wave propagation in heterogeneous media. Comput. Methods Appl. Mech. Eng., 193:4837-4856, 2004.

[26] J. Fish, W. Chen, and G. Nagai. Non-local dispersive model for wave propagation in heterogeneous media: one-dimensional case. Int. J. Numer. Methods Eng., 54:331-346, 2002a.

[27] J. Fish, W. Chen, and G. Nagai. Non-local dispersive model for wave propagation in heterogeneous media: multi-dimensional case. Int. J. Numer. Methods Eng., 54:347-363, 2002b.

[28] G. Floquet. Sur les équations différentielles linéaires à coefficients périodiques. Ann. École Norm. Sup, $12: 47-88,1883$.

[29] Gaston Floquet. Sur les équations différentielles linéaires à coefficients périodiques. 12:47-88, 1883. 
[30] L. Guillot, Y. Capdeville, and J. J. Marigo. 2-D non-periodic homogenization of the elastic wave equation: SH case. Geophysical Journal International, 182(3):1438-1454, 2010.

[31] B.B. Guzina, S. Meng, and O. Oudghiri-Idrissi. A rational framework for dynamic homogenization at finite wavelengths and frequencies. Proceedings of the Royal Society A, 475:20180547, 2019.

[32] Duc Trung Le and Jean-Jacques Marigo. Second order homogenization of quasi-periodic structures. Vietnam Journal of Mechanics, 40(4):325-348, 2018.

[33] Q. Ma and J. Cui. Second-order two-scale analysis method for the quasi-periodic structure of composite materials under condition of coupled thermo-elasticity. Adv. Mater. Res., 629:160-164, 2013.

[34] M. Maldovan. Sound and heat revolutions in phononics. Nature, 503:209-217, 2013.

[35] S. Meng and B. Guzina. On the dynamic homogenization of periodic media: Willis' approach versus two-scale. Proc. R. Soc. A, 474(20170638), 2018.

[36] G. Milton, M. Briane, and J. Willis. On cloaking for elasticity and physical equations with a transformation invariant form. New J. Phys., 8:248-267, 2006.

[37] G.W. Milton and J.R. Willis. On modifications of newton's second law and linear continuum elastodynamics. Proc. R. Soc. A, 463:855-880, 2007.

[38] Y. Miyamoto, W.A. Kaysser, B.H. Rabin, A. Kawasaki, and R.G. Ford. Functionally Graded Materials: Design, Processing and Applications, volume 5. Springer Science \& Business Media, 2013.

[39] S. Moskow and M. Vogelius. First-order corrections to the homogenised eigenvalues of a periodic composite medium. a convergence proof. Proceedings of the Royal Society of Edinburgh Section A: Mathematics, 127:1263-1299, 1997.

[40] H. Nassar, Q.C. He, and N. Auffray. Willis' elastodynamic homogenization theory revisited for periodic media. J. Mech. Phys. Solids, 77:158-178, 2015.

[41] M. Ruzzene and A. Baz. Control of wave propagation in periodic composite rods using shape memory inserts. J. Vib. Acoust., 122:151-159, 2000.

[42] E. Sánchez-Palencia. Non-homogeneous Media and Vibration Theory, volume 127. Lecture Notes in Physics, 1980.

[43] M. Silva. Study of pass and stop bands of some periodic composites. Acta. Acust., 75(1):62-68, 1991.

[44] F. Su, J.Z. Cui, Z. Xu, and Q.L. Dong. Multiscale method for the quasi-periodic structures of composite materials. Appl. Math. Comput., 217:5847-5852, 2011. 
[45] K.L. Tsakmakidis, A.D. Boardman, and O. Hess. "trapped rainbow" storage of light in metamaterials. Nature, 450:397-401, 2007.

[46] A. Wautier and B. Guzina. On the second-order homogenization of wave motion in periodic media and the sound of a chessboard. J. Mech. Phys. Solids, 78:382-414, 2015.

[47] Z. Yang, Y. Sun, J. Cui, and X. Li. A multiscale algorithm for heat conduction-radiation problems in porous materials with quasi-periodic structures. Commun. Comput. Phys., 24(1):204-233, 2018.

[48] J Zhu, Y Chen, X Zhu, FJ Garcia-Vidal, X Yin, W Zhang, and X Zhang. Acoustic rainbow trapping sci, 2013.

[49] J. Zhu, J. Christensen, J. Jung, L. Martin-Moreno, X. Yin, L. Fok, X. Zhang, and F. J. Garcia-Vidal. A holey-structured metamaterial for acoustic deep-subwavelength imaging. Nat. Phys., 7(52-55), 2011. 